\title{
Labor Force Participation and Pair-wise Efficient Contracts with Search and Bargaining
}

\author{
Bryan Engelhardt* \\ David L. Fuller \\ College of the Holy Cross Concordia University
}

January 20, 2012

\begin{abstract}
A "constant" wage is pair-wise inefficient in a standard search model when workers endogenously separate from employment. We derive a pair-wise efficient employment contract that involves workers paying a hiring fee (or bond) upon the formation of a match. We estimate the constant wage and pair-wise efficient contract assuming the hiring fee is unobservable, and find evidence to reject the pair-wise efficient contract in favor of the constant wage rule. A counterfactual experiment reveals the current level of labor force participation to be $9.6 \%$ below the efficient level, and a structural shift to the pair-wise efficient contract improves welfare by roughly $3.5 \%$.
\end{abstract}

Keywords: labor supply, unemployment, matching, efficiency wages

JEL Codes: J0, J41, J64

*One College Street, Worcester, MA 01610 USA; (508)793-3669; bengelha@ holycross.edu. 


\section{Introduction}

Transitions in and out of the labor force represent an important component of labor market activity. The movement of individuals from employment to non-participation represents one example of these transitions. For the U.S., we find these transitions are significant and vary across the wage distribution, with those at the lower end exiting at a faster rate than those at the higher end. Specifically, the National Longitudinal Survey of Youth (NLSY), which we use in this paper, indicates that transitions from employment to non-participation represent the most common transition employed individuals make; they occur more frequently than transitions to a new job or to unem-

ployment. Despite the importance of these flows for labor market outcomes, the literature studying equilibrium labor markets has devoted little attention to labor market participation decisions, and even less to the transition from employment to non-participation. Recent work in this area, while limited, has developed along two dimensions: one attempting to develop models to explain the transitions into and out of non-participation, and another that includes these transitions in policy experiments, allowing researchers to determine the effect of policies on the composition of the labor force.

We develop a model that contributes to the literature on both fronts. First, our model is capable of explaining how the transition from employment to non-participation changes over the wage distribution, a feature that remains relatively unexplored. Second, we find that when building a model to explain these flows, the policy implications remain unclear. Specifically, our analysis characterizes a potentially large negative externality arising from a worker's choice to transition from employment to non-participation. After recognizing the potential inefficiency, we determine if U.S. data remains consistent with employment contracts that internalize the externality, or with standard employment contracts that do not. Thus, the outcome of our analysis has important implications for the efficacy of labor market policies, especially those directed towards altering the composition of the labor force. In other words, we ask the question: Does the current level of labor force participation maximize welfare? If the answer is no, then what are the potential welfare gains from adopting alternative employment contracts?

To capture the large transitions between labor market states, we extend the search-matching model in Engelhardt, Rocheteau \& Rupert (2008). The model has an endogenous labor force participation decision where individuals receive idiosyncratic shocks affecting the value of non- 
market activities. Our extension of the model includes match specific productivity, resulting in a distribution of wages, transition rates that vary conditional on the wage, and thus transition rates depending on the employment contract determining the wage.

Modeling the decision to exit employment, however, introduces theoretical complications. Specifically, when agents take actions affecting the duration of the match, the nature of the employment contract affects the efficiency properties of the models' equilibria. In exploring this fact, we demonstrate what a pair-wise efficient employment contract looks like, and show that a standard surplus splitting rule, or constant wage, is not pair-wise efficient.

The endogenous transition from employment to non-participation implies pair-wise inefficiency because it introduces a negative externality. In our model, while employed in a productive match, individuals receive opportunities for non-market activities. In some periods, the value of accepting these opportunities exceeds the value of retaining the match, and as a result, an individual may decide to quit and leave the labor force. The externality arises because individuals fail to internalize the cost the separation imposes on their firm, who paid a fixed cost to post the vacancy and hire the worker. When the employment contract consists of only a constant wage, determined by a surplus splitting rule, the aforementioned externality obtains; therefore, any equilibria produced by the model fail to be pair-wise efficient. This occurs because ameliorating the externality requires firms to pay a wage equal to the productivity of the match. Of course, when the employment contract consists of only a constant wage, no active equilibrium retains this feature. We derive an alternative employment contract, one where workers receive a wage equal to the productivity of the match and pay a "hiring fee" to the firm upon the formation of the match. The introduction of the hiring fee allows the externality to be internalized and pair-wise efficiency is achieved.

In this paper, we focus on determining if the pair-wise efficient contract or the constant wage rule is more consistent with U.S. data. To make this comparison, we estimate both models using wage and duration data, assuming the hiring fee is unobservable. From the estimates, we compare the fit of the two models. While we do not measure an actual hiring fee (or other features of wage contracts resembling one, e.g. wage-tenure contracts) in the data, the two models do predict different combinations of wages and durations. Thus, despite not explicitly observing a hiring fee, observed wages and durations could be consistent with the model featuring such wage contracts. The idea is to determine whether the data remain more consistent with the implications of the 
pair-wise efficient contract or the inefficient constant wage rule.

With NLSY data, we estimate both models using maximum likelihood estimation. We find that under certain parametric restrictions, the likelihood function that incorporates the pair-wise efficient contract is nested within the likelihood function that features the inefficient constant wage rule. Given this fact, we are then able to perform a formal specification test to determine which contract best describes the U.S. data. The results indicate that the model with an inefficient constant wage rule provides a better fit than the model with the pair-wise efficient contract.

Given evidence of a pair-wise inefficient contract, we then quantify its consequences. Specifically, we perform a counterfactual exercise to determine the welfare gains of a structural shift from the constant wage contract to the pair-wise efficient one. We find labor force participation would rise by $9.6 \%$, employment by $17.9 \%$, and unemployment would fall $10.8 \%$. Overall, the adoption of the pair-wise efficient contract increases welfare by $3.5 \%$.

This paper contributes to several strands of the labor literature, and in particular the research on labor market search. ${ }^{1}$ First, several authors (for example Stevens (2004), Shimer (2005a), and Bonilla \& Burdett (2005)) show that if workers take actions affecting the duration of the match, then inefficiencies can arise depending on the nature of the employment contract. Menzio \& Shi (2011) also examine a model with on the job search, focusing on cyclical issues. These papers focus on job-to-job transitions, and develop alternative bargaining structures that admit efficient separations. In contrast, we focus on transitions from employment to non-participation, and compare the pair-wise efficient contract to an inefficient one. While several authors have attempted to estimate similar search models with on-the-job-search, to the best of our knowledge, our paper represents the first attempt to empirically test two competing wage structures. Also related, Albrecht, Navarro \& Vroman (2010) construct a model with endogenous participation decisions and workers who are heterogenous with respect to their market productivity. They show that in this environment, the Hosios rule (Hosios (1990)) does not imply efficiency; instead, it implies labor force participation remains too high, or that too many vacancies are created in equilibrium.

A few authors have also attempted to incorporate a labor market decision into search models. Gautier, Moraga-Gonzalez \& Wolthoff (2009) include labor force participation in a model with variable search intensity. Similarly to our results, they find the existing labor market equilibrium

\footnotetext{
${ }^{1}$ The literature on search models of the labor market is extensive; consequently, we only discuss those papers most closely related to ours.
} 
in the Netherlands to be inefficient, and recommend changes to minimum wage and unemployment insurance policies to move closer to the socially optimal outcome. Garibaldi \& Wasmer (2005) develop a search model with stochastic participation costs to analyze the labor force participation decision. In their model however, the wage distribution remains degenerate; as a result, it is unable to capture the flow of employed workers to non-participation across the wage distribution. Pries \& Rogerson (2004) also develop a search model with matching frictions to study labor force participation decisions. Their model focuses on the dynamic nature of participation decisions, and qualitatively captures the correlation between participation rates and participation flows. Our model extends their analysis along two dimensions. First we perform a general equilibrium analysis, allowing for job creation decisions by firms. Second, we allow for a continuous distribution of idiosyncratic shocks to the relative value of non-participation. Pries \& Rogerson (2004) use a two-shock case, which does not capture the variation in exit rates across the wage distribution. Finally, our work is also related to Yip (2003), who develops and estimates a search model with endogenous labor force participation decisions, driven by idiosyncratic shocks to the relative value of non-participation. Yip (2003) performs some counterfactual policy experiments, but does not derive Pareto optimal allocations. The counterfactual exercises in Yip (2003) focus on how changes in policy variables (such as unemployment benefits) affect the composition of the labor market as well as welfare. In contrast, we focus first on determining which employment contract best describes the data, and our counterfactual exercise calculates the gains from achieving pair-wise efficiency.

This work also contributes to the literature investigating the role of various labor market policies. Flinn (2006) represents one recent example of this discussion. Flinn analyzes the role of minimum wage policies in a search model with matching frictions similar to the one we use here. The focus of his analysis rests on determining whether the Hosios condition (Hosios (1990)) holds when the parameters are estimated using U.S. data. When the resulting parameters do not satisfy the aforementioned condition, the paper suggests there may exist a role for minimum wage policies in improving welfare. In contrast, our analysis abstracts from inefficiencies arising from parametric restrictions, focusing instead on structural inefficiencies. Flinn \& Mabli (2008) represents another example studying the effects of minimum wage policies in a similar model with on-the-job search. This work is similar to ours in that the specific employment contract affects the welfare properties of equilibria. 
Finally, the paper contributes to the efficiency wage literature (Carmichael (1990) contains a critical survey). For instance, we find firms pay higher wages due to job turnover. However, we do not find efficiency wages because of a strict assumption about the job turnover to wage relationship. $^{2}$ Rather, we deduce higher wages due to the increased bargaining position of the worker. More importantly, we contribute to the bonding critique. The pair-wise efficient contract we study involves workers paying a "hiring" fee upon the formation of the match; this represents a similar concept to the "bonding" approach used in the efficiency wage literature. Much of that literature has assumed bonds do not exist and argued for theoretical reasons why this might be true. In contrast, we assume the bonds are unobservable and test for their presence.

The remainder of the paper proceeds as follows. Section 2 introduces the environment. Section 2.2 describes the individual(household) and firm decision problems, and Section 2.3 characterizes the resulting equilibrium. Section 3 outlines the data, the estimation procedure, and discusses the results. Finally, Section 4 discusses the counterfactual welfare exercises while Section 5 explores the robustness of our results. All proofs are presented in Appendix A.

\section{Model}

\subsection{The Environment}

The economy consists of a unit-measure of infinitely-lived individuals and a large measure of firms. Time, $t$, is continuous and goes on forever. Each individual has an endowment of one indivisible unit of time with three alternative, mutually exclusive uses: search for a job, work for a firm, or enjoy leisure (i.e. do not participate in the formal labor market). Individuals and firms are risk-neutral and discount at rate $r>0$.

An unemployed individual searching for a job enjoys utility flow $b$, interpreted as the utility from not working. Upon receiving a job offer when unemployed, individuals and firms observe a match specific productivity, $y$, drawn from a distribution $F(y)$ with support $[0, \bar{y}]$, and decide whether to accept or reject the match. Firms are composed of a single job, either filled or vacant, and discount future profits at rate $r$. Vacant firms are free to enter and pay a flow cost, $\gamma>0$, to advertise a vacancy. Vacant firms produce no output and filled firms produce the same final good.

\footnotetext{
${ }^{2}$ This could be assumed and would result in even higher wages.
} 
The labor market is subject to matching frictions. The aggregate matching function, $\zeta(U, V)$, describes the flow of job offers, where $U$ represents the measure of unemployed workers actively looking for jobs, and $V$ the measure of vacant jobs. The matching function, $\zeta$, is continuous, strictly increasing, strictly concave (with respect to each of its arguments), and exhibits constant returns to scale. Furthermore, $\zeta(0, \cdot)=\zeta(\cdot, 0)=0$ and $\zeta(\infty, \cdot)=\zeta(\cdot, \infty)=\infty$. Following Pissarides' terminology, define $\theta \equiv V / U$, referred to as labor market "tightness." Each vacancy is filled according to a Poisson process with arrival rate $\frac{\zeta(U, V)}{V} \equiv q(\theta)$. Similarly, each unemployed worker finds a job according to a Poisson process with arrival rate $\frac{\zeta(U, V)}{U}=\theta q(\theta)$. Filled jobs receive negative idiosyncratic productivity shocks rendering the match unprofitable with a Poisson arrival rate $s$. Denote by $n_{e}(y), n_{u}$, and $n_{o}$ the measure of workers employed with productivity $y$, unemployed, and not in the labor force, respectively.

The existence of opportunities to exit the labor force represents an important feature of our model. When an individual decides to enter non-participation, she enjoys an instantaneous flow utility from leisure, $\varepsilon$, which remains stochastic. ${ }^{3}$ When employed and unemployed, with arrival rate $\lambda_{e}$ and $\lambda_{u}$, respectively, the individual draws a new leisure value from the distribution $G(\varepsilon)$, and decides whether to enjoy the leisure and enter non-participation, or remain in the current state. Once in non-participation, she enjoys constant flow utility $x$. With arrival rate $\lambda_{o}$, the individual draws a new value of $\varepsilon$ from the distribution $G(\varepsilon)$. If the individual decides to enjoy the leisure, she remains in non-participation; if she does not, then she uses her time endowment searching, and thus transitions to unemployment.

\subsection{Bellman equations}

We consider two different employment contracts. The first contract specifies that upon the formation of a match, the worker pays a hiring fee, $\phi$, and then receives a constant wage, $w$, thereafter. As in Engelhardt et al. (2008), this contract is Pareto-optimal, and we refer to it as the "pair-wise efficient contract." Alternatively, we consider an employment contract featuring only a wage determined by an exogenous surplus splitting rule, similar to the one used in Pissarides (2000). In either case, $w$, or the pair $(\phi, w)$, are determined through a bargaining solution. ${ }^{4}$ This section begins

\footnotetext{
${ }^{3}$ Alternatively, one could interpret the instantaneous flow utility as coming from home production.

${ }^{4}$ Implicit in this formulation is the firm commits to the terms of the employment contract. In particular, once the worker pays the hiring fee the firm does not renege on the promised future wage.
} 
with the case of the pair-wise efficient contract. We focus on steady-state equilibria, where market tightness, $\theta$, matching probabilities, and exit rates remain constant over time. In the next two subsections, we describe the flow Bellman equations for individuals and firms, and characterize the employment contracts.

\subsubsection{Individuals}

Denote the value of being an individual in state $i \in\{u, e, o\}$ by $\mathscr{V}_{u}, \mathscr{V}_{o}$ and $\mathscr{V}_{e}(y)$, respectively. An individual in state $i=u$ is considered unemployed, $i=e$ employed, and $i=o$ not in the labor force (non-participation). The flow Bellman equations for individuals' value functions follow

$$
\begin{aligned}
r \mathscr{V}_{u} & =b+\theta q(\theta) \int\left[\mathscr{V}_{e}(y)-\mathscr{V}_{u}-\phi(y)\right]^{+} d F(y)+\lambda_{u} \int\left[\varepsilon+\mathscr{V}_{o}-\mathscr{V}_{u}\right]^{+} d G(\varepsilon) \\
r \mathscr{V}_{e}(y) & =w(y)+s\left(\mathscr{V}_{u}-\mathscr{V}_{e}(y)\right)+\lambda_{e} \int\left[\varepsilon+\mathscr{V}_{o}-\mathscr{V}_{e}(y)\right]^{+} d G(\varepsilon) \\
r \mathscr{V}_{o} & =x+\lambda_{o} \int \max \left\{\varepsilon, \mathscr{V}_{u}-\mathscr{V}_{o}\right\} d G(\varepsilon)
\end{aligned}
$$

where $[z]^{+}=\max (z, 0)$.

An individual accepts a job offer if $w \geq w\left(y_{R}\right)$, where $y_{R}$ defines the point where workers remain indifferent between becoming employed or remaining unemployed, which satisfies

$$
V_{e}(y)-\phi(y) \geq V_{e}\left(y_{R}\right)-\phi\left(y_{R}\right)=V_{u} .
$$

From (1), (2), and (3) an individual in state $i$ chooses to exit the labor market whenever $\varepsilon \geq \varepsilon_{i}$ where

$$
\begin{aligned}
\varepsilon_{u} & =\mathscr{V}_{u}-\mathscr{V}_{o} \\
\varepsilon_{o} & =\mathscr{V}_{u}-\mathscr{V}_{o} \\
\varepsilon_{e}(y) & =\mathscr{V}_{e}(y)-\mathscr{V}_{o}
\end{aligned}
$$

From (5)-(7) the value of the opportunity that makes an individual in a given state indifferent between exiting or not, $\varepsilon_{i}$, is equal to the expected cost of leaving, $\mathscr{V}_{e}(y)-\mathscr{V}_{0}$. Further notice that $\varepsilon_{o}=\varepsilon_{u}$. Subtracting (5) from (7), we have 


$$
\mathscr{V}_{e}(y)-\mathscr{V}_{u}=\varepsilon_{e}(y)-\varepsilon_{u}
$$

\subsubsection{Firms}

Firms participating in the market can be in one of two states: they either hold a vacant job $(v)$ or a filled job $(f)$. The following summarize firms' flow Bellman equations.

$$
\begin{aligned}
r \mathscr{V}_{v} & =-\gamma+q(\theta) \int_{y_{R}}\left(\phi(y)+\mathscr{V}_{f}(y)-\mathscr{V}_{v}\right) d F(y) \\
r \mathscr{V}_{f}(y) & =y-w(y)-s\left(\mathscr{V}_{f}(y)-\mathscr{V}_{v}\right)-\lambda_{e}\left[1-G\left(\varepsilon_{e}\right)\right]\left(\mathscr{V}_{f}(y)-\mathscr{V}_{v}\right)
\end{aligned}
$$

Free-entry of firms implies $\mathscr{V}_{v}=0$ and therefore, from (9),

$$
\int_{y_{R}}\left(\phi(y)+\mathscr{V}_{f}(y)\right) d F(y)=\frac{\gamma}{q(\theta)}
$$

Note, if $\lambda_{e}=\lambda_{u}=\lambda_{o}=0$ and $F(y)$ remains degenerate, the model is exactly Pissarides (2000).

\subsubsection{Employment contract with a hiring fee}

Engelhardt et al. (2008) show that in a model with this type of exit from employment, employed workers separate "too frequently" because they do not internalize the negative externality they impose on the firm. They also show that by allowing the employment contract to include the upfront fee, $\phi(y)$, the worker and the firm can reach a pair-wise efficient outcome. ${ }^{5}$ The employment contract $(\phi, w)$ is determined by the generalized Nash solution, where $\beta \in(0,1)$ denotes the worker's bargaining power. Specifically, the contract satisfies

$$
(\phi, w)=\arg \max \left(\mathscr{V}_{e}(y)-\mathscr{V}_{u}-\phi(y)\right)^{\beta}\left(\mathscr{V}_{f}(y)-\mathscr{V}_{v}+\phi(y)\right)^{1-\beta}
$$

The following Lemma describes the properties of the Pareto optimal, or pair-wise efficient employment contract.

\footnotetext{
${ }^{5}$ Alternatively, the optimal contract could take the form of a constant wage, $w(y)$, and a payment from the worker to the firm (a fine) if the worker exits. This transfer would exactly compensate the firm for its lost surplus, and is equivalent to the current contract.
} 
Lemma 1 The pair-wise efficient contract is

$$
\begin{aligned}
& w(y)=y, \\
& \phi(y)=(1-\beta)\left(\mathscr{V}_{e}(y)-\mathscr{V}_{u}\right) .
\end{aligned}
$$

According to Lemma 1, the pair-wise efficient contract sets the wage equal to the worker's productivity. ${ }^{6}$ To fully describe the equilibrium in the next section, it is useful to write the first order condition of (12) with respect to $\phi(y)$ :

$$
(1-\beta)\left[\mathscr{V}_{e}(y)-\mathscr{V}_{u}-\phi(y)\right]=\beta\left[\mathscr{V}_{f}(y)+\phi(y)\right]
$$

While the contract described above is pair-wise efficient, there is also the issue of efficient entry of vacancies. Specifically, in the canonical model of Pissarides (2000), Hosios (1990) shows that efficiency along this dimension is achieved when the bargaining parameter, $\beta$, is equated with the elasticity of the matching function. While this applies to the basic search model with matching frictions, Albrecht et al. (2010) show that in a model with endogenous labor market entry, imposing the Hosios condition leads to excessive vacancy creation, and efficiency along the entry margin is not achieved. Thus, it may be the case that in our model, imposing the Hosios condition does not lead to efficiency. Although we do not analytically characterize the efficient matching function elasticity, in our sensitivity analysis in Section 5 we do examine this issue.

\subsubsection{Alternative modeling structures and wage determination mechanisms}

There exist many alternative wage contracts and bargaining games that obtain pair-wise efficiency. For example, we could allow workers and firms to renegotiate after the worker receives an outside opportunity. In this case, the firm would continue to increase the wage offer until profits become zero and the match dissolves as the individual transitions to non-participation. It also remains possible that wage contracts have a wage-tenure feature, so that wages increase a specified amount based on the duration of the match. To determine whether the data remains consistent with the pairwise efficient contract or not, however, does not depend on the specific contract, per se. Instead, it only depends on how well the wages and durations predicted by the model match with those in the

\footnotetext{
${ }^{6}$ Since the firm makes no profit after the hiring fee has been paid, it has no incentive to fire the worker as the value of a vacancy is no greater than the value of a filled job, i.e., $\mathscr{V}_{f}(y)=\mathscr{V}_{v}=0$.
} 
data. To the extent that these alternative wage contracts admit pair-wise efficient separations, they remain observationally equivalent.

In the case of renegotiated wages (or wage-tenure contracts), there are some differences, which could affect the results of the estimation. For example, in the aforementioned wage determination schemes, if these contracts obtained in the data, one would observe wages increasing over the duration of the match. While our hiring fee model and this renegotiated wage model both obtain the efficient exit rates, the hiring fee model predicts a constant wage over the duration of a match. While allowing for renegotiation upon receipt of an outside option is appealing theoretically, there exists evidence that suggests this practice is relatively uncommon. Bowlus \& Neumann (2005) examine the determinants of wage growth, and specifically analyze the relative importance of wage growth over the duration of a match. They find "that the on-the-job wage growth component is a small fraction of overall wage growth, which suggests that job mobility may be the most important component in earnings growth," (Bowlus \& Neumann (2005), p.218). We further address the issue of job-to-job transitions in Section 5. Given the evidence, we argue that our pair-wise efficient contract formulation remains the most reasonable among the alternatives, and provides the best starting point for comparing against the inefficient contract.

\subsubsection{Constant wage employment contract}

We now consider the case of a constant (or exogenous) wage. In this case, we assume the same objective function as equation (12), but add the constraint that $\phi(y)=0$. With this assumption, we restrict the wage contract to consist only of the wage payment. Thus, in this case, wages are determined according to

$$
w(y)=\arg \max \left(\mathscr{V}_{e}(y)-\mathscr{V}_{u}\right)^{\beta}\left(\mathscr{V}_{f}(y)\right)^{1-\beta}
$$

The F.O.C. of this objective imply the following surplus splitting equation

$$
(1-\beta)\left[\mathscr{V}_{e}(y)-\mathscr{V}_{u}\right]=\beta\left(\mathscr{V}_{f}(y)\right)
$$

which corresponds to the standard search and bargaining surplus splitting wage equation, as discussed in Pissarides (2000). While this surplus splitting equation represents the unique arg max of the aforementioned objective, it does not represent a Nash Bargaining solution. Details of this fact 
are described in detail in Shimer (2005a).

Now, using (8), (17), and

$$
\mathscr{V}_{f}(y)=\frac{y-w(y)}{r+s+\lambda_{e}\left[1-G\left(\varepsilon_{e}(y)\right)\right]}
$$

we can write the wage in this case as

$$
w(y)=y-\frac{1-\beta}{\beta}\left(r+s+\lambda_{e}\left[1-G\left(\varepsilon_{e}(y)\right)\right]\right)\left(\varepsilon_{e}(y)-\varepsilon_{u}\right)
$$

Note, since $\beta \in(0,1), y>w(y)$ and from (18) $\mathscr{V}_{f}(y)>0$, this contract does not achieve pair-wise efficiency (does not maximize surplus of a match).

\subsection{Equilibrium}

This section characterizes the equilibrium for the aforementioned economy. To facilitate the presentation of both models simultaneously, we introduce an indicator function, $\chi$, where $\chi=1$ when the employment contract includes the hiring fee, $\phi(y)$, and $\chi=0$ for the constant wage case. The idea is to show the equilibrium can be represented by three equations in three unknowns, $\varepsilon_{e}(y), \varepsilon_{u}$, and $\theta$. Towards this end, the free entry condition implies

$$
\frac{\gamma}{q(\theta)}=\int_{y_{R}}\left(\mathscr{V}_{f}(y)+\chi \phi(y)\right) d F(Y)
$$

Using (15) for the pair-wise efficient contract case, and (17) for the constant wage contract case, to solve for $\mathscr{V}_{f}(y)$, substituting into (20) gives

$$
\frac{\gamma}{q(\theta)}=\frac{(1-\beta)}{\beta}\left(\int_{y_{R}}\left(\mathscr{V}_{e}(y)-\mathscr{V}_{u}-\chi \phi(y)\right) d F(Y)\right) .
$$

Next, using (3), (5), (6), and integrating by parts we can write

$$
r V_{o}=x+\lambda_{o}\left[\varepsilon_{u}+\int_{\varepsilon_{u}}[1-G(\varepsilon)] d \varepsilon\right]
$$

Then, combining (1), (3), (5), (20), (22), and integrating by parts, we have

$$
\left(r+\lambda_{o}\right) \varepsilon_{u}=b-x+\frac{\beta \gamma \theta}{(1-\beta)}+\left(\lambda_{u}-\lambda_{o}\right) \int_{\varepsilon_{u}}[1-G(\varepsilon)] d \varepsilon
$$


This provides the first relationship between $\varepsilon_{e}(y), \varepsilon_{u}$, and $\theta$. Similarly, for $y \geq y_{R}$, using (2), (7), (8), and integration by parts, we have

$$
\begin{aligned}
& r \varepsilon_{e}(y)+\lambda_{o}\left[\varepsilon_{u}+\int_{\varepsilon_{u}}[1-G(\varepsilon)] d \varepsilon\right]+x \\
& =\chi y+(1-\chi) w(y)+s\left(\varepsilon_{u}-\varepsilon_{e}(y)\right)+\lambda_{e} \int_{\varepsilon_{e}(y)}[1-G(\varepsilon)] d \varepsilon
\end{aligned}
$$

where for $\chi=0, w(y)$ follows (19). Equations (23) and (24) determine the values of $\varepsilon_{u}$ and $\varepsilon_{e}(y)$ as functions of $\theta$. Then, using (1), (2), and (21), we can write

$$
\begin{aligned}
& \int_{y_{R}}\left\{\chi y+(1-\chi) w(y)-b-\frac{\beta \theta \gamma}{1-\beta}+\lambda_{e} \int_{\varepsilon_{e}(y)}[1-G(\varepsilon)] d \varepsilon\right. \\
& \left.-\lambda_{u} \int_{\varepsilon_{u}}[1-G(\varepsilon)] d \varepsilon\right\} d F(y)-\frac{(r+s) \gamma}{(1-\beta) q(\theta)}=0
\end{aligned}
$$

Thus, equations (23)-(25) determine the equilibrium values of $\varepsilon_{u}, \varepsilon_{e}(y)$, and $\theta$.

To complete the description of equilibrium, consider the steady state distribution of individuals across states, $\left(n_{e}(y), n_{u}, n_{o}\right)$, where $n_{i}$ denotes the number of individuals in state $i \in\{u, e, o\}$. This distribution is characterized by the following three equations.

$$
\begin{gathered}
s \int_{y_{R}} n_{e}(y) d F(y)+n_{o} \lambda_{o} G\left(\varepsilon_{o}\right)=\left\{\theta q(\theta)\left[1-F\left(y_{R}\right)\right]+\lambda\left[1-G\left(\varepsilon_{u}\right)\right]\right\} n_{u} \\
\theta q(\theta)\left[1-F\left(y_{R}\right)\right] f\left(y \mid y \geq y_{R}\right) n_{u}=\left(\lambda\left[1-G\left(\varepsilon_{e}(y)\right)\right]+s\right) n_{e}(y), \forall y \in\left[y_{R}, \bar{y}\right] \\
\int_{y_{R}} n_{e}(y) d F(y)+n_{u}+n_{o}=1
\end{gathered}
$$

Figure 1 illustrates these flows.

We can now define an equilibrium for the model.

Definition 1 A steady state equilibrium is a list, $\left\{\theta, \varepsilon_{u}, \varepsilon_{e}(y), n_{e}(y), n_{u}, n_{o}\right\}$, such that $\theta, \varepsilon_{u}$, and $\varepsilon_{e}(y)$ solve (21), (23), and (24); and $n_{e}(y), n_{u}, n_{o}$ solve (26)-(28).

Determining an equilibrium represents a fixed point problem in $\theta$. Specifically, for an initial value of $\theta,(23)$ and (24) determine values for $\varepsilon_{u}$ and $\varepsilon_{e}(y)$. Given these values, (21) determines a new value of $\theta$. This process continues until (21) holds. In general, a unique equilibrium may not 
Figure 1: Worker Flows

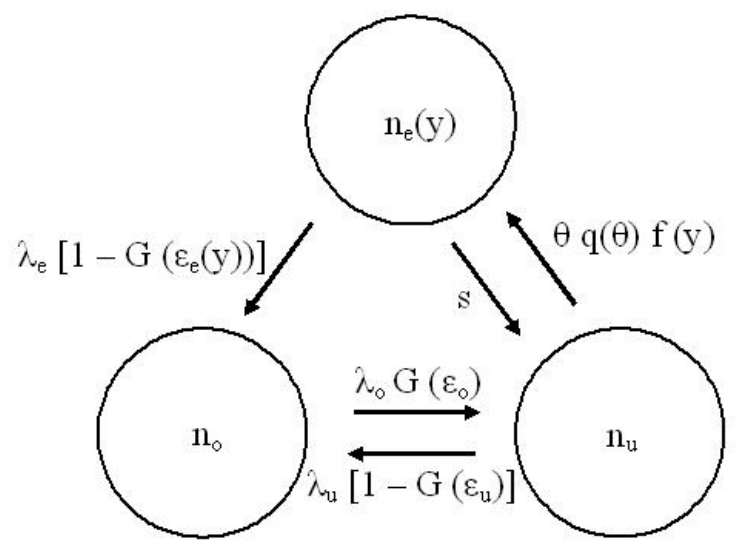

exist without further restrictions on the parameter space, or the functional forms of $G$ and $F$. Below we summarize these restrictions in the following results, where we define $h(x)=\int_{x}[1-G(\varepsilon)] d \varepsilon$.

Lemma 2 If $\lambda_{e} \geq \lambda_{u}$, and $G(\varepsilon)$ is such that $h(x)$ is convex, then an active equilibrium exists for both $\chi=0$ and $\chi=1$.

Thus, under mild restrictions on $G(\varepsilon)$ and the parameters $\lambda_{e}$ and $\lambda_{u}$, an equilibrium exists in both wage contract models. To reiterate, refer to Appendix A for all proofs.

Further restrictions are necessary for uniqueness.

Proposition 1 If $\lambda_{e} \geq \lambda_{u} \geq \lambda_{o}, \chi=1$, and $G(\varepsilon)$ is such that $h(x)$ is convex, then there exists $a$ unique equilibrium.

In general, finding restrictions ensuring uniqueness of the equilibrium in the constant wage contract case is more difficult. One simple restriction is $\lambda_{e}=0$; however, in this case, the comparison of the two wage contracts is less interesting. Below is another possible parametric restriction.

Proposition 2 If $\lambda_{e} \geq \lambda_{u} \geq \lambda_{o}, \chi=0, G(\varepsilon)$ is such that $h(x)$ is convex, and if for a given $\hat{y}$ and $A$ it is true that $f(y) \leq A \forall y \geq \hat{y}$, then there exists a unique equilibrium. 
Intuitively, this states that there exists a unique equilibrium if the productivity distribution $F(y)$ does not put too much weight on higher values of $y$. In the simulations presented below, the sufficient conditions for existence of an equilibrium are satisfied. Our parametrization also satisfies the sufficient conditions of Proposition 1. The sufficient conditions of Proposition 2 remain more difficult to verify, but we found the equilibrium always appeared to be unique. Specifically, we performed the aforementioned fixed point problem for a "low" and "high" starting value of $\theta$. In all cases we tested, the operator converged to the same equilibrium value of $\theta$.

To this point, we have described two models, each of which makes predictions about labor market behavior and outcomes. Given the specified employment contract, each model has equilibrium flows into and out of the various labor market states, as well as the steady state levels of employment and labor market participation. The question we now pursue is which model best describes the behavior observed in the U.S. labor market? To perform this comparison, we estimate each model, and compare their respective predictions with observed behavior.

\section{Estimation}

We use Maximum Likelihood Estimation (MLE) to identify the parameters for each model specification. In this section we describe the data, derive the likelihood function, discuss identification, and provide the estimates of the two models' parameters. With these estimates, we then perform a likelihood ratio test to determine which model remains more appropriate for describing observed behavior.

\subsection{Data}

The data comes from the National Longitudinal Survey 1997 (NLSY97), a panel data set of individuals in the U.S. between the ages of 12 and 16 in 1997. The NLSY97 observes when an individual is employed, unemployed, or out of the labor force, in addition to an individual's wage if employed. We estimate each model from the duration and wage data.

We start the sample on January 1, 2002; as a result, the individuals we observe are at least seventeen years of age. We follow Wolpin (1987) and Bowlus, Kiefer \& Neumann (1995) in determining who should be included in the sample. Specifically, we limit the sample to individuals 
who have exited school with less than a college degree; that is, we remove from the sample those who have not yet graduated from school and are still enrolled. We also remove observations with missing wage data and/or missing labor force status. Aside from the aforementioned cases, we include all observations in our sample.

To analyze the sample, we adopt a stock sampling approach. Specifically, we record what state an individual is initially in and record the duration of time spent in the original state (unemployed, employed or out of the labor force), where the time spent is $t_{i}$ for $i=\{e, u, o\}$. After a span of time, an individual transitions from the original state " $i$ " to the new state " $j$." Let $N_{i, j}=1$ if an observation started in state $i$ and transitioned to state $j$ and zero otherwise, where $i, j \in\{e, u, o\} .^{7}$ Finally, the wage $w_{e}$ is observed for those whom are initially employed as well as the wage $w_{u}$ for those whom transition from unemployment to employment (wages are recorded as the hourly wage). Table 1 contains the descriptive statistics. Figure 2 presents the duration data graphically. The wage data are presented graphically in Section 3.5 when we compare the fit of the efficient and inefficient contracts against the observed data.

Table 1: Descriptive Statistics

\begin{tabular}{lcccccc} 
& \multicolumn{2}{c}{ Men \& Women } & \multicolumn{2}{c}{ Men } & \multicolumn{2}{c}{ Women } \\
\cline { 2 - 6 } Variable & Mean & Std. Dev. & Mean & Std. Dev. & Mean & Std. Dev. \\
\hline Transitions from $u$ to $o\left(N_{u, o}\right)$ & 0.043 & 0.202 & 0.042 & 0.202 & 0.043 & 0.202 \\
Transitions from $u$ to $e\left(N_{u, e}\right)$ & 0.055 & 0.229 & 0.066 & 0.247 & 0.044 & 0.205 \\
Transitions from $o$ to $u\left(N_{o, u}\right)$ & 0.31 & 0.463 & 0.303 & 0.46 & 0.319 & 0.466 \\
Transitions from $e$ to $u\left(N_{e, u}\right)$ & 0.321 & 0.467 & 0.348 & 0.476 & 0.291 & 0.455 \\
Transitions from $e$ to $o\left(N_{e, o}\right)$ & 0.259 & 0.438 & 0.227 & 0.419 & 0.294 & 0.456 \\
Duration Unemployed $\left(t_{u}\right)$ & 1.363 & 1.644 & 18.005 & 22.897 & 17.329 & 19.091 \\
Duration Outside Labor Force $\left(t_{o}\right)$ & 2.537 & 3.625 & 28.77 & 43.548 & 37.499 & 50.345 \\
Duration Employed $\left(t_{e}\right)$ & 3.872 & 4.459 & 51.659 & 61.198 & 48.841 & 54.093 \\
Wage if initially employed $\left(w_{e}\right)$ & 8.858 & 4.147 & 9.457 & 4.471 & 8.185 & 3.637 \\
Wage if inititally unemployed $\left(w_{u}\right)$ & 8.542 & 4.431 & 9.064 & 4.916 & 7.659 & 3.309 \\
Observations & \multicolumn{2}{c}{3593} & & 1908 & & 1685 \\
\hline
\end{tabular}

Note: Durations are quarterly and wage statistics are hourly. Transitions do not sum to one due to right censoring and the wage distribution is trimmed at the $2.5 \%$ level. Missing wage and duration data is assumed to occur randomly and are excluded.

\footnotetext{
${ }^{7}$ If we observe an individual transition from out of the labor force to employment, we assume they are unemployed for a period of time less than the frequency of the data, which is a week.
} 
Figure 2: Duration of Employment, Unemployment, and Outside the Labor Force
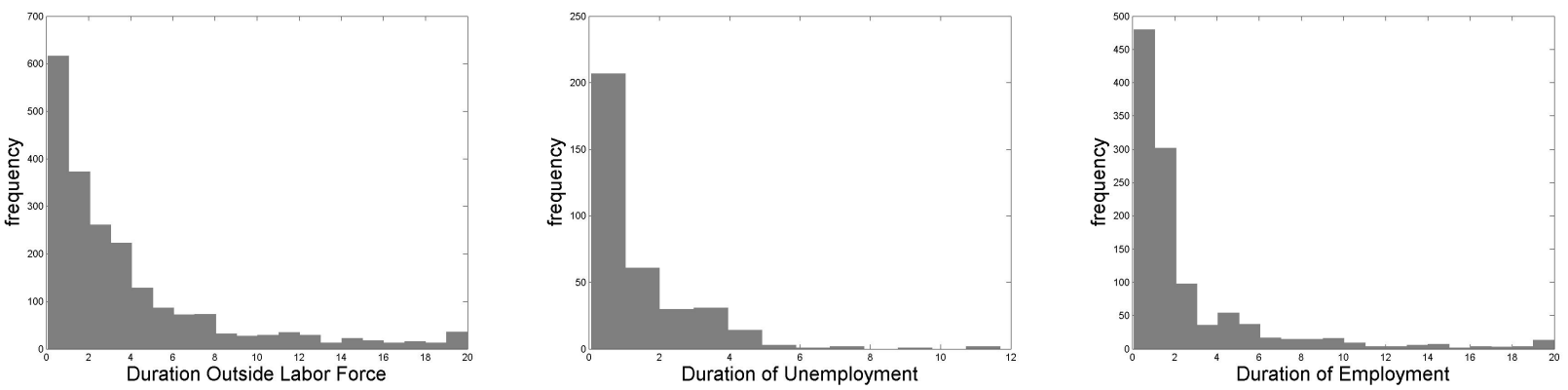

\subsection{Likelihood Function, constant wage employment contract}

We begin by presenting the estimation of the constant wage employment contract. The likelihood function consists of three multiplicative components derived from those we initially observe to be employed, unemployed, or not participating. In constructing the likelihood function, each component includes the length of time individuals spend in the initial state and the likelihood of being in their initial state. Once individuals transition, we observe and use the information on where they transitioned. For those employed at some point during the sample, we observe and incorporate their wage into the likelihood function.

Non-participants represent a straightforward component of the likelihood function. In particular, individuals transition according to a Poisson process with rate $\delta=\lambda_{o} G\left(\varepsilon_{o}\right)$. As a result, an individual's duration of time outside the labor force has an exponential distribution. Refer to Chapter 5 in Lancaster (1990) for the derivation of the probability distribution from stock sampling. Given this, non-participants contribute to the likelihood function as

$$
P\left(N_{o, u}, t_{o}\right)=n_{o} \delta e^{-\delta t_{o}}
$$

where $n_{o}$ is the probability of being outside the labor force and is a function of the Poisson arrival rates as described in the flow equations (26)-(28).

The unemployed contribute to the likelihood function in three ways. Given they are initially unemployed with probability $n_{u}$, their duration of unemployment factors in as

$$
f_{u}\left(t_{u} \mid u\right)=\left\{h_{u}+k_{u}\right\} e^{-\left\{h_{u}+k_{u}\right\} t_{u}},
$$

where $h_{u}=\theta q(\theta)\left[1-F\left(y_{R}\right)\right]$ and $k_{u}=\lambda_{u}\left[1-G\left(\varepsilon_{u}\right)\right]$. Second, they contribute the probability of 
transitioning to employment or non-participation as

$$
\begin{aligned}
& P\left(N_{u, e} \mid u\right)=\frac{h_{u}}{h_{u}+k_{u}}, \\
& P\left(N_{u, o} \mid u\right)=\frac{k_{u}}{h_{u}+k_{u}} .
\end{aligned}
$$

Finally, if they transition to employment, then they draw a match specific productivity and in turn wage according to

$$
f_{w}\left(w_{u} \mid N_{u, e}\right)=\frac{\partial y\left(w_{u}\right)}{\partial w_{u}} \frac{f\left(y\left(w_{u}\right)\right)}{1-F\left(y_{R}\right)} .
$$

where the productivity of the match, $y\left(w_{u}\right)$, is derived from (19). The unemployed's complete contribution to the likelihood function is

$$
P\left(t_{u}, N_{u, e}, N_{u, o}, w_{u}\right)=n_{u} f_{u}\left(t_{u} \mid u\right) P\left(N_{u, o} \mid u\right)^{N_{u, o}}\left[P\left(N_{u, e} \mid u\right) f_{w}\left(w_{u} \mid N_{u, e}\right)\right]^{N_{u, e}} .
$$

The employed contribute to the likelihood function in two ways. Given they are initially employed and paid $w_{e}$ with probability $n_{e}\left(y\left(w_{e}\right)\right)$, their duration of employment enters as

$$
f_{e}\left(t_{e} \mid e, w_{e}\right)=\left\{s+k_{e}\left(y\left(w_{e}\right)\right)\right\} e^{-\left\{s+k_{e}\left(y\left(w_{e}\right)\right)\right\} t_{e}},
$$

where $k_{e}\left(y\left(w_{e}\right)\right)=\lambda_{e}\left[1-G\left(\varepsilon_{e}\left(y\left(w_{e}\right)\right)\right)\right]$. Second, their probability of transitioning to unemployment or out of the labor force is

$$
\begin{aligned}
P\left(N_{e, u} \mid e, w_{e}\right) & =\frac{s}{s+k_{e}\left(y\left(w_{e}\right)\right)}, \\
P\left(N_{e, o} \mid e, w_{e}\right) & =\frac{k_{e}\left(y\left(w_{e}\right)\right)}{s+k_{e}\left(y\left(w_{e}\right)\right)} .
\end{aligned}
$$

As a result, the complete contribution of the employed is

$$
P\left(t_{e}, N_{e, u}, N_{e, o}, w_{e}\right)=n_{e}\left(y\left(w_{e}\right)\right) f_{e}\left(t_{e} \mid e, w_{e}\right) P\left(N_{e, u} \mid e, w_{e}\right)^{N_{e, u}} P\left(N_{e, o} \mid e, w_{e}\right)^{N_{e, o}},
$$

The complete likelihood function can thus be written as ${ }^{8}$

$$
\ln L=\sum \ln \left(P\left(N_{o, u}, t_{o}\right)\right)+\sum \ln \left(P\left(t_{u}, N_{u, e}, N_{u, o}, w_{u}\right)\right)+\sum \ln \left(P\left(t_{e}, N_{e, u}, N_{e, o}, w_{e}\right)\right)
$$

\footnotetext{
${ }^{8}$ Although not described in (29), right censoring has been accounted for in the usual manner.
} 
where the sums, from left to right, represent the observations that start in the o, $\mathrm{u}$, and e state, respectively. Since the distributions $G(\varepsilon)$ and $F(y)$ will take on an assumed parametric form, they can be characterized by the parameter vectors $\tau_{g}$ and $\tau_{f}$, respectively.

\subsection{Identification, constant wage contract}

This section describes identification of the constant wage contract case. Identification of the pairwise efficient contract is similar and described in the next section.

The likelihood function in (29) leaves the following parameters to be determined: $s, \lambda_{o}, \lambda_{u}$, $\lambda_{e}, r, b, x, \gamma, \beta, \tau_{g}, \tau_{f}$, and the parameters of the matching function. Ignoring the parameters that determine $n_{o}, n_{u}$, and $n_{e}(y)$, identification of $\delta, s, h_{u}$ and $k_{u}$ follows directly from the first order conditions of the likelihood function. More specifically, the simple exponential form for the duration data implies

$$
\begin{aligned}
\delta & =\frac{\bar{N}_{o, u}}{\bar{t}_{o}}, \\
s & =\frac{\bar{N}_{e, u}}{\bar{t}_{e}}, \\
k_{u} & =\frac{\bar{N}_{u, o}}{\bar{t}_{u}}, \\
h_{u} & =\frac{\bar{N}_{u, e}}{\bar{t}_{u}},
\end{aligned}
$$

where $\bar{N}_{i, j}$ and $\bar{t}_{i}$ is the mean of $N_{i, j}$ and $t_{i}$, respectively. Although $\delta, k_{u}, h_{u}$ are not parameters of interest, they are used to deduce $\lambda_{o}, \lambda_{u}, x$ and the parameters of the matching function, which we discuss in more detail below. Estimating $n_{o}, n_{u}$, and $n_{e}(y)$ directly is a simple multinomial problem. We use more robust estimates, however, by substituting in the steady state probability of being in each state as defined by the flow equations.

We take a reduced-form approach to estimating $k_{e}\left(y\left(w_{e}\right)\right)$ (which we use to infer $\left.\lambda_{e}\right)$, fitting it according to $\kappa_{1} \exp \left(\kappa_{2} w\right)$. Specifically, we plug $\hat{k}_{e}(w)=\kappa_{1} \exp \left(\kappa_{2} w\right)$ into (29) and maximize the likelihood with respect to $\kappa_{1}$ and $\kappa_{2}$. Note, if we find $\kappa_{2}>0$, then individuals exit at a faster rate the more they earn. Alternatively, if $\kappa_{2}<0$, then the more individuals earn the less likely they are 
to exit their job. This approach is similar to Bontemps, Robin \& van den Berg (2000) where they use a non-parametric procedure to estimate the wage distribution, which they then use to deduce other parameters from the model. We have also tried a nonparametric procedure, where we use a constant rate of exit, $k_{e}(w)=\kappa_{i}$, for fixed intervals of the wage distribution, and found similar results to our reduced-form approach. Comparison of the model's exit rates and the reduced-form estimates are provided in Figure 4 below.

We use the wage distribution to identify the parameter vector $\tau_{f}$. In doing so, a complication arises as $y\left(w_{u}\right)$ and $y\left(w_{e}\right)$ are functions of the difference $\varepsilon_{e}(y)-\varepsilon_{e}\left(y_{R}\right)$ as seen in (19). To circumvent this difficulty, we assume the distribution of outside opportunities is exponential, so $G(\varepsilon)=1-\exp \left(-\frac{1}{\mu_{g}} \varepsilon\right)$. Under this parametric assumption, the difference can be derived in a relatively straightforward manner. First, from (2) and (7) we can write

$$
(r+s)\left(\varepsilon_{e}(y)-\varepsilon_{e}\left(y_{R}\right)\right)=(r+s)\left(\mathscr{V}_{e}(y)-\mathscr{V}_{e}\left(y_{R}\right)\right)=w(y)-y_{R}+\lambda_{e}\left[-\int_{\varepsilon_{e}\left(y_{R}\right)}^{\varepsilon_{e}(y)}[1-G(\varepsilon)] d \varepsilon\right]
$$

then from the distributional assumption on $G(\varepsilon)$, (30) can be further simplified to

$$
\varepsilon_{e}(y)-\varepsilon_{e}\left(y_{r}\right)=\frac{w-y_{R}+\left(k_{e}(y(w))-k_{e}\left(y_{R}\right)\right) \mu_{g}}{r+s} .
$$

As a result, plugging (31) into (19), and substituting in the reduced-form estimates $\hat{k}_{e}\left(w_{i}\right)$ and $\hat{k}_{e}\left(y_{R}\right)$, we can write the following:

$$
y_{i}=w_{i}+\alpha\left(\frac{w_{i}-y_{R}+\left(\hat{k}_{e}\left(w_{i}\right)-\hat{k}_{e}\left(y_{R}\right)\right) \mu g}{r+s}\right)\left[r+s+\hat{k}_{e}\left(w_{i}\right)\right],
$$

for $i \in\{u, e\}$ and $\alpha=(1-\beta) / \beta$. We refer to the inefficient wage splitting rule as defined by (32) as Specification $I$.

Given the difference in $\varepsilon_{e}(y)$ and $\varepsilon_{e}\left(y_{R}\right)$, we are able to discuss identifying the wage distribution. Due to the issues discussed in Flinn \& Heckman (1982), we are required to assume a parametric distribution for wages and use the log-normal as it is the standard, ${ }^{9}$ or

$$
f_{w}\left(w_{i}\right)=\frac{\partial y\left(w_{i}\right)}{\partial w_{i}} \frac{1}{y\left(w_{i}\right) \sigma_{f} \sqrt{2 \pi}} \exp \left\{-\frac{1}{2}\left(\frac{\ln y\left(w_{i}\right)-\mu_{f}}{\sigma_{f}}\right)^{2}\right\} /\left(1-F\left(y_{R}\right)\right),
$$

\footnotetext{
${ }^{9}$ The Weibull has also been used with similar results.
} 
for $i \in\{u, e\}$.

Although we do not provide a proof of identification for $\kappa_{1}, \kappa_{2}, \alpha, \mu_{g}, \mu_{f}$, and $\sigma_{f}$, we have run Monte Carlo experiments to test our optimization procedure. We find 1500 observations is sufficient in providing reliable estimates for the aforementioned parameters. In other words, we simulate data from the model, plug the data into likelihood function, and estimate the parameters. The results show the parameters used to generate the data are identified with the likelihood function even though the function can have all the standard issues when maximizing a function of many variables.

Given the estimates from the likelihood function and by imposing three additional identifying restrictions, we are able to determine the remaining parameters $\lambda_{o}, \lambda_{u}, \lambda_{e}, x, b$, the matching function parameters, and $\gamma$ using the model's equilibrium conditions. The restrictions are for a time period of one quarter. So, we set $r=0.01$, consistent with a risk-free interest rate of $4 \%$ per annum. We also assume $\lambda_{o}=\lambda_{u}$ and the Hosios condition $(\beta=\eta)$, where $\eta$ is defined by the Cobb-Douglas matching function $m(u, v)=A u^{\eta} v^{1-\eta} .{ }^{10}$ We investigate the impact of changes in $\eta$ in Section 5.

To determine the remaining parameters, note $\alpha$ is estimated by the likelihood function, thus $\beta$ follows directly from $\alpha=(1-\beta) / \beta$. Given $\beta$, we determine $\eta$ from the Hosios condition. In determining the remaining parameters of the matching function, we follow Engelhardt et al. (2008) and recognize $\theta$ can be normalized to one, given $\gamma$ remains free to be used to equate the free entry of firms, and $\theta q(\theta)$ has been identified. Hence, we set $\theta=1$ and deduce $A=h_{u} /\left(1-F\left(y_{R}\right)\right)$ as the right hand side is estimated by the likelihood function.

To obtain $\lambda_{o}, \lambda_{u}$ and $\lambda_{e}$, we take the assumption $\lambda_{o}=\lambda_{u}$ and the fact $\varepsilon_{o}=\varepsilon_{u}$ to determine the function $\frac{\delta}{\kappa_{u}}=\frac{G\left(\varepsilon_{u}\right)}{\left(1-G\left(\varepsilon_{u}\right)\right)}$ which implies a value for $\varepsilon_{u}$. Plugging $\varepsilon_{u}$ back into the definitions of $\delta$ and $\kappa_{u}$ we get values for $\lambda_{o}$ and $\lambda_{u}$ given the estimate of $\mu_{g}$. Finally, we use the fact $\lambda_{e} / \lambda_{u}=$ $k(e)\left(y_{R}\right) / k_{u}=\lambda_{u} /\left(\kappa_{1} \exp ^{\kappa_{2} \min \left(w_{e}\right)}\right) / k_{u}$ to deduce $\lambda_{e}$ as $V_{e}\left(y_{R}\right)=V_{u} \cdot{ }^{11}$

Finally, we plug the known parameters into the reservation wage equation, $V_{e}\left(y_{R}\right)=V_{u}$, to deduce $b$, use the equilibrium condition (23) to determine $x$, and use the free-entry condition

\footnotetext{
${ }^{10}$ The Hosios condition can be relaxed if a CRS matching function is assumed or JOLTS data is incorporated as discussed by Engelhardt \& Rupert (2011).

${ }^{11}$ It is possible to estimate $\lambda_{e}(y)$. However, we take a more conventional approach and assume one exit rate.
} 


$$
\gamma=q(\theta) \int_{y_{R}} \frac{y-w(y)}{r+s+\lambda_{e}\left(1-G\left(\varepsilon_{e}(y)\right)\right)} f(y) d y
$$

to estimate $\gamma$.

\subsection{Estimation of the pair-wise efficient contract}

Estimation of the pair-wise efficient contract follows the estimation of the constant wage contract. Specifically, if $\alpha=0$, then the pair-wise efficient contract's likelihood function is identical to the one with the constant wage. In other words, the likelihood function in (29) remains unchanged except that now $w_{i}=y_{i}$ for $i \in\{u, e\}$. To reiterate, the new wage equation is obtained by setting $\alpha=0$ in equation (32). As a result, the likelihood function with the pair-wise efficient contract is nested within the likelihood function with the inefficient contract. To put it differently, the pairwise efficient contract's likelihood function is a constrained version of the constant wage contract's likelihood function. Results from the constant likelihood function, or estimates of the pair-wise efficient contract, are denoted as Specification $E$.

Our ability to nest the two likelihood functions and thus perform a formal specification test depends on our reduced-form approach to estimating $k_{e}(y)$. Under this assumption, the exit rate is given by $\kappa_{1} \exp \left(\kappa_{2} w\right)$ for both contracts; however, calculating $k_{e}(y)$ from the model's equilibrium conditions would result in different functions, $\varepsilon_{e}(y)$, and thus the likelihood functions would not be nested. This can be seen from equation (24). While the reduced-form approach does represent a simplification, it does not appear to affect the results. In Figure 4, the left of the two plots compares the reduced-form estimates of $k_{e}(y)$ against those implied in the model (for the parameters estimated under Specification I). From this figure it can be seen that the reduced-form approximates the more complicated function arising from the model. To put it another way, we are using the wage distribution, and not the exit rates, to compare the two models.

\subsection{Model comparison}

We have developed and estimated two models of the U.S. labor market. The analysis now focuses on evaluating how well each model explains the observed outcomes. The comparison is strengthened by the fact that the pair-wise efficient contract is nested within the inefficient contract. If 
$\alpha=0$, then the likelihood functions of the two models coincide; therefore, if the constraint $\alpha=0$ is rejected in favor of $\alpha \neq 0$, then we have evidence in favor of the inefficient contract.

Before going further, we stress the comparison is made by examining the fit of each models' predicted wage distribution to the observed wage distribution. While in theory it would be interesting to incorporate additional types of data including any up-front fees charged to the employee, the cost of forgone wages due to a probationary period, or penalties from quitting such as the loss of a retirement plan, it remains practically impossible as these variables are difficult to measure. Moreover, the inclusion of the hiring fee can have many alternative interpretations; it remains equivalent to a "quitting" fee, or could be interpreted as capturing some wage-tenure features of employment contracts, such as increasing wages. Therefore, one of the key contributions of this paper is to be able to deduce the type of contract with a limited amount of information. In other words, the important question is not whether we observe a hiring fee (or a "quitting" fee, etc.). Rather, what matters is whether the observed wages, durations, and transitions remain consistent with individuals internalizing the negative externality. If observed wages, transitions, and durations are consistent with the efficient exit rates, then we would fail to reject the hypothesis that $\alpha=0$. Therefore, we do not need to observe the specific employment contract to make this comparison.

Table 2 presents the results from the estimation of the two likelihood functions under their respective specifications using the entire sample, estimates using a sub-sample made up exclusively of men, and estimates using a sub-sample made up exclusively of women. We have included estimates for men and women separately as any study of labor force participation decisions should account for potential differences between them. Given the differences, it is feasible that the results are affected by treating both groups equally and in one market. Therefore, to check the robustness of our results, we separate the sample into males and females and perform the analysis on each group independently as well as jointly. In estimating the groups separately, we follow van den Berg \& Ridder (1998), where heterogeneity is introduced "by assuming that there are separate labor markets (or segments of the labor market, or sub-markets) for different types of individuals," (van den Berg \& Ridder (1998) p. 1203). In doing so, we are able to compare the differences in transition rates and productivity parameters. In addition, we analyze whether either group exits efficiently.

In general, we find the unemployed exit the labor force at one-quarter the rate they enter employment. Also, those in the lower quartile of the wage distribution exit employment for non- 


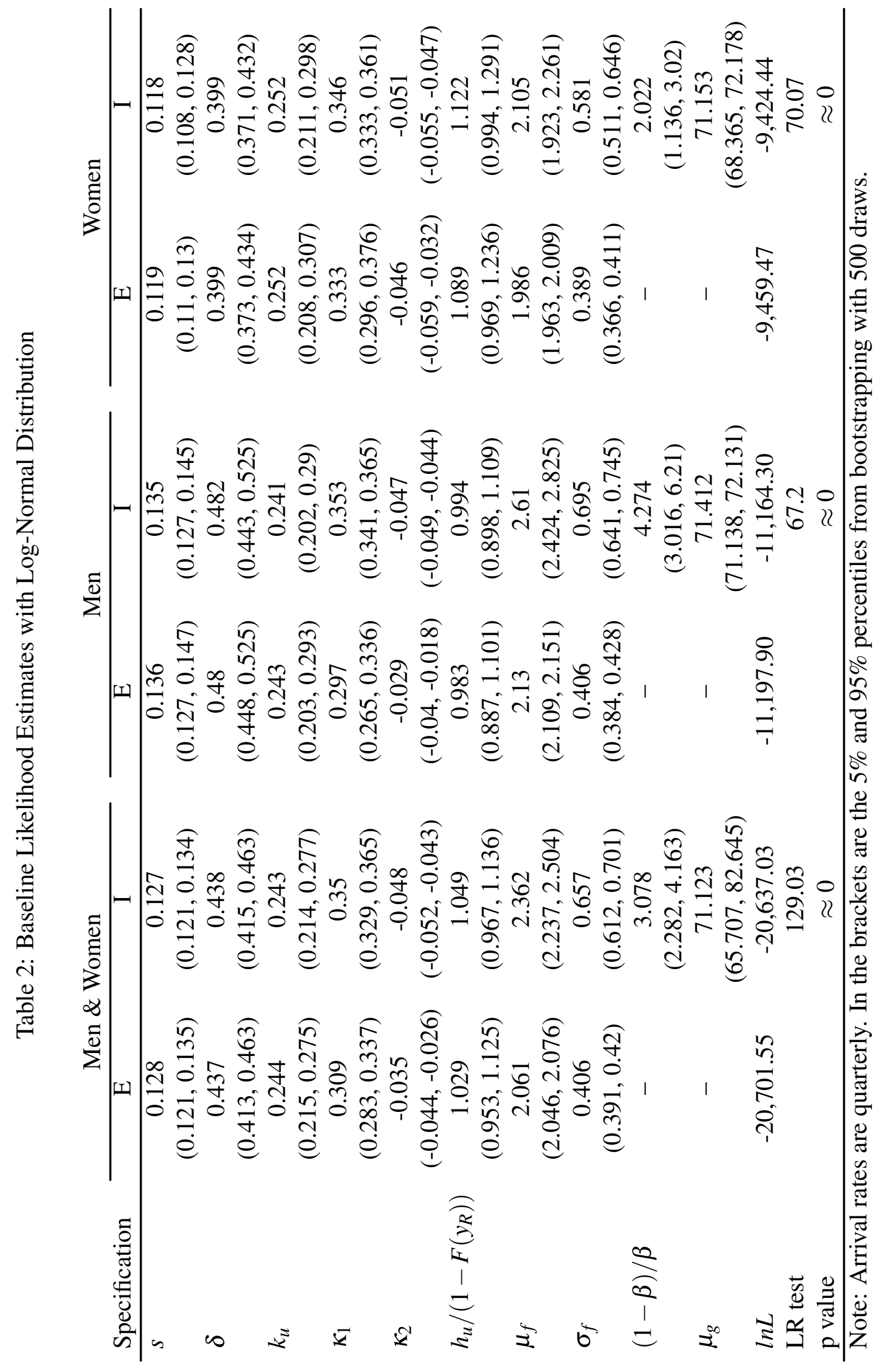


participation $20 \%$ faster than those in the upper quartile. Note the transition rates remain relatively high compared to other studies; however, our sample consists of young individuals who experience higher transition rates. In particular, the exit rate $\delta$ states the average amount of time spent outside the labor force is 2.28 quarters. To some, this may seem high. However, the average duration outside the labor force $\left(t_{o}\right)$ is 2.5 quarters (the estimate of $\delta$ is slightly different due to stock sampling and right censoring). Figure 2 provides a closer look at $t_{o}$. In terms of the male and female comparison, we note the transition rates are similar. The estimates show females transition to non-participation at a slightly faster rate while they transition into the labor force from nonparticipation at a slower rate. Furthermore, the female productivity estimates are lower while the bargaining parameter is slightly higher.

For our analysis, $\alpha$ represents the key parameter. Recall, in our formulation of the likelihood function, the value of the likelihood function under the inefficient contract must be greater than the value of the likelihood function of the pair-wise efficient contract. This occurs because the likelihood functions only differ in terms of the wage distributions, and the wage distributions remain identical if $\alpha=0$; therefore the likelihood function of the pair-wise efficient contract is nested in the likelihood function of the inefficient contract. This connection between the two likelihood functions allows for a clear comparison based on the fit of the wage distribution, and more specifically allows for a formal specification test (a likelihood ratio test). Regarding the fit, the likelihood ratio test, presented at the bottom of Table 2, rejects the pair-wise efficient contract in favor of the inefficient contract. This is true whether we estimate the likelihood function with the entire sample or for men and women separately.

To understand the results of the statistical test, we plot the key difference between the efficient and inefficient estimates in Figure 3. This figure compares the two wage distributions for the entire sample (wages offered at the time of hire $\left(w_{u}\right)$ and employed wage distribution $\left(w_{e}\right)$ ) observed in the data against the predicted distributions. Although the distinctions are subtle in the figure, the inefficient contract fits the distribution better than the pair-wise efficient contract. In particular, the inefficient contract has a larger mass of individuals at the lower end of the distribution, matching a noticeable feature of the observed distribution.

Analytically, this arises from the second term in (32). If the term was constant or a linear function of $w$, then the inefficient and efficient distributions would be hard to differentiate as the location and scale parameters of the productivity distribution would compensate this difference. 
Therefore, the key is the way in which the last term of (32) changes. In particular, the term grows with $y$, implying the proportion of the surplus going to workers shrinks as $y$ grows. This occurs because the rates of exit, $k_{e}(y)$, fall as output, $y$, and wages, $w$, grow. As a result, the inefficient contract produces a larger weight at the lower end of the distribution. Intuitively, the individuals are losing their ability to bargain as productivity increases because their outside option is becoming less appealing. Therefore, the inefficient contract predicts that the wage rises as productivity rises, but at a slower rate. Given this, the inefficient contract has less dispersion relative to the pair-wise efficient contract, in addition to predicting the larger mass of individuals in the lower end of the distribution.

Figure 3: Offered and Employed Hourly Wage Distributions - Men \& Women
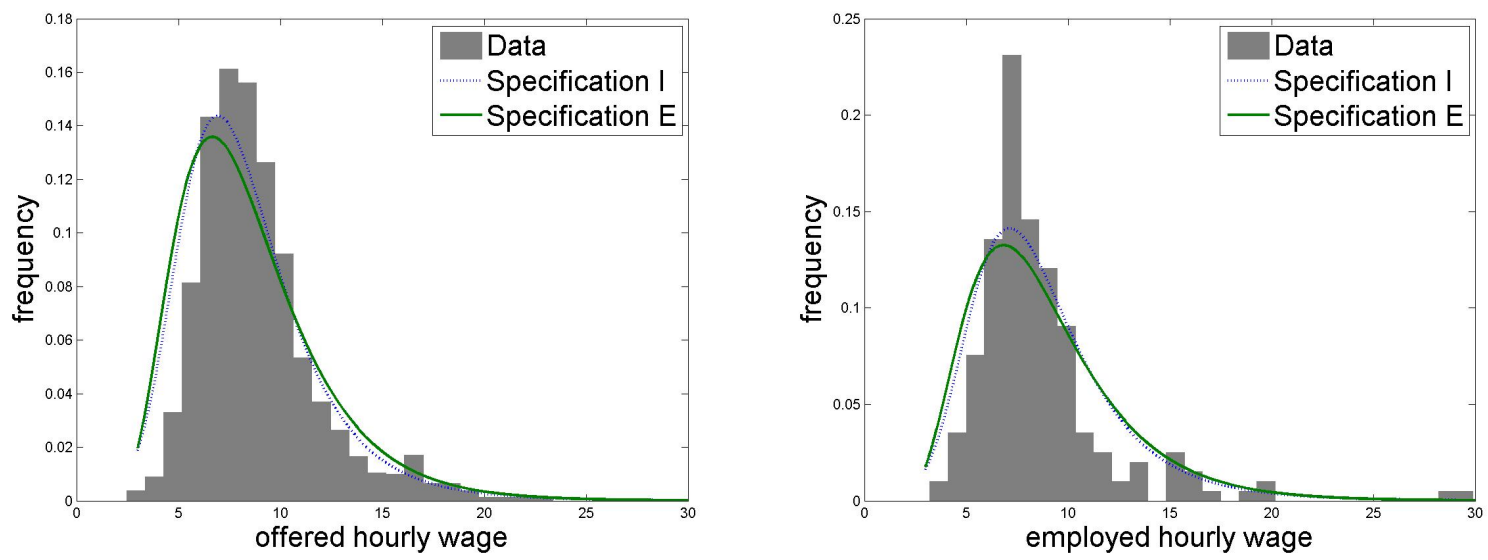

Note: The Savitzky-Golay smoothing filter with degree three has been applied to the data for display purposes only.

In the next section, we explore the differences in welfare between the constant wage contract and the pair-wise efficient contract. Given the employment contract featuring only a constant wage best describes the data, we use the parameters from Specification $I$ (discussion of the identification of the estimates is provided in Section 3.3). Table 3 presents the list of the parameters and their values for the whole sample as well as men and women separately.

It is also useful to compare our estimates of some parameters to those found in the existing literature. It may appear $b$ and $x$ are low, which, when compared to Hagedorn \& Manovskii (2008), for example, they are. However, in the relevant literature, these values have a relatively wide range of estimates. The most closely related model to ours, Flinn (2006), estimates $b$ using wage data, and finds similar values to ours. Furthermore, the relatively low value of $x$ reflects how the model 
Table 3: Model Parameters

\begin{tabular}{lcccl}
\hline & Men \& Women & Men & Women & \\
$r$ & 0.01 & 0.01 & 0.01 & discount rate \\
$b$ & 0.541 & 0.932 & -0.286 & unemployed utility flow \\
$x$ & -43.363 & -49.824 & -38.442 & outside the labor force utility flow \\
$A$ & 1.049 & 0.994 & 1.122 & efficiency of matching function \\
$\eta$ & 0.245 & 0.19 & 0.331 & elasticity of matching function \\
$\beta$ & 0.245 & 0.19 & 0.331 & bargaining power of workers \\
$\gamma$ & 20.54 & 28.853 & 13.106 & recruiting cost \\
$s$ & 0.127 & 0.135 & 0.118 & job destruction rate \\
$\mu_{f}$ & 2.362 & 2.61 & 2.105 & mean of log normal distribution of job productivity \\
$\sigma_{f}$ & 0.657 & 0.695 & 0.581 & s.d. of log normal distribution of job productivity \\
$\mu_{g}$ & 71.123 & 71.412 & 71.153 & mean of exponential distribution of outside options \\
$\lambda_{u}$ & 0.681 & 0.723 & 0.651 & unemployed flow of outside utility \\
$\lambda_{o}$ & 0.681 & 0.723 & 0.651 & outside labor force flow of outside utility \\
$\lambda_{e}$ & 0.847 & 0.92 & 0.767 & employed flow of outside utility \\
\hline
\end{tabular}

encompasses utility outside the labor force; it is the sum of $x$ and the random shocks $\varepsilon$. Once these shocks are included, the utility from outside the labor force is very close to b. In terms of our estimate for $\gamma$, it is roughly three times output while the related calibration literature estimates it to be a half or less. That being said, our estimates are lower than the most closely related work of Flinn (2006). Finally, we note our estimate of $\beta$ is in the middle of the wide range of estimates. It is lower than in Shimer (2005b), larger than Hagedorn \& Manovskii (2008) find, and it is relatively close to Flinn (2006).

\section{Welfare \& efficiency}

Based on the analysis thus far, we have concluded that the constant wage contract represents the more appropriate model for capturing the observed labor market equilibrium. To quantitatively assess the implications of this, we investigate the available welfare gains of moving to the pairwise efficient equilibrium. To accomplish this, we perform the following counterfactual exercise. We take the parameters estimated for the constant wage contract, and calculate the equilibrium of the model using the pair-wise efficient contract. From these calculations, we compare how the levels of unemployment, employment, labor force participation, and ultimately welfare would 
change if the market moved from the inefficient contract to the efficient one.

To perform the counterfactual exercise, we use the following welfare function

$$
\begin{aligned}
W= & n_{u}\left(b+\lambda_{u} \int_{\varepsilon_{u}} \varepsilon d G(\varepsilon)-\theta \gamma\right)+\int_{y_{R}}\left\{n_{e}(y)\left[y+\lambda_{e} \int_{\varepsilon_{e}(y)} \varepsilon d G(\varepsilon)\right]\right\} d y \\
& +n_{o}\left(x+\lambda_{o} \int_{\varepsilon_{o}} \varepsilon d G(\varepsilon)\right) .
\end{aligned}
$$

From Equation 33, welfare equals the sum of unemployed workers' consumption $(b)$, the average output $(y)$ produced in each match, the utility flow of those of non-participants $(x)$, and the utility from taking the outside options and either exiting the labor force or staying outside the labor force, minus the recruiting expenses incurred by firms to find unemployed workers $(\gamma){ }^{12}$

Table 4 presents the increase in welfare and the change in labor market outcomes, given a structural shift from a constant wage contract to the pair-wise efficient contract. From Table 4, labor force participation would rise by $9.6 \%$, unemployment would decrease by $10.8 \%$, and employment would increase by $17.9 \%$. When employed individuals internalize the negative externality imposed by their decisions to quit, they exit at a slower rate than in the constant wage contract. As a result, labor force participation rises. Also, the number of vacancies opened by firms increases as matches become longer and more valuable. Therefore, we observe a lower unemployment rate as well. Overall, we see a shift to the pair-wise efficient contract would result in an increase in welfare of $3.5 \%$ in the "men \& women" sample. The gains are larger for males as their productivity is estimated to be higher and thus the cost of an inefficient separation is higher.

In terms of the pair-wise inefficiency, the rate employed individuals exit the labor force is the key. It also represents the primary difference between the pair-wise efficient employment contract and the contract featuring only a constant wage. The rate at which an individual exits the labor force from employment is given by $\lambda_{e}\left[1-G\left(\varepsilon_{e}(w(y))\right)\right]$. One prediction of this model is whether exit rates from employment to non-participation vary across the distribution of wages. Since $\varepsilon_{e}(w(y))$ increases with the wage, depending on the distribution of $G(\varepsilon)$, employed individuals in the upperend of the wage distribution may exit at a slower rate than those in the lower end of the distribution. Indeed, we observe different exit rates over the wage distribution. Figure 4 provides the reducedform exit rates given by the likelihood function. They are plotted by wages and output. In addition,

\footnotetext{
${ }^{12}$ The number of vacancies in equilibrium is equal to the product of market tightness, $\theta$, and the measure of unemployed workers, $n_{u}$.
} 
Table 4: Labor Market Outcomes from Shift in Contract

\begin{tabular}{rccc} 
Parameters & $\begin{array}{c}\text { Estimated } \\
\text { Contract } \\
\text { (Inefficient) }\end{array}$ & $\begin{array}{c}\text { Counterfactual } \\
\text { Contract } \\
\text { (Efficient) }\end{array}$ & $\begin{array}{c}\text { Percentage } \\
\text { change }\end{array}$ \\
\cline { 2 - 3 } Not in Labor Force (\%) & 37.8 & Men \& Women & \\
Unemployed (\%) & 17.6 & 15.7 & -15.87 \\
Employed (\%) & 44.6 & 52.6 & -10.8 \\
Welfare & 24.35 & 25.21 & 3.94 \\
& & $\underline{\text { Men }}$ & \\
Not in Labor Force (\%) & 35.6 & 27.1 & -23.88 \\
Unemployed (\%) & 19.1 & 15.4 & -19.37 \\
Employed (\%) & 45.3 & 57.5 & 26.93 \\
Welfare & 24.79 & 26.62 & 7.38 \\
& & $\underline{\text { Women }}$ & \\
Not in Labor Force (\%) & 40.1 & 36.4 & -9.23 \\
Unemployed (\%) & 16 & 15.2 & -5 \\
Employed (\%) & 44 & 48.4 & 10 \\
Welfare & 23.94 & 24.24 & 1.25 \\
\hline
\end{tabular}

it displays the rate workers exit under each contract assuming Specification $I$ and the parameter estimates it implies are correct. Thus, the pair-wise efficient contract estimates that are displayed in the figure are the counterfactual results found when running the model with the parameter estimates from Specification $I$.

Figure 4 is important for two reasons. First, it provides support that the exit rates from employment to non-participation decline as wages rise, highlighting the importance of including a non-degenerate productivity distribution in the model. Second, internalizing the cost of exiting represents the key to improving welfare. If one looks at the exit rates by the output of a job, then the pair-wise efficient contract implies a much smaller exit rate as workers internalize the full cost of exiting. However, it might be surprising that workers exit at a slightly faster rate under the pairwise efficient contract when looking at it by the wage. The reason is in the inefficient contract, the wage does not coincide with the output of a job as it does in the case of the pair-wise efficient contract. Moreover, in the inefficient contract, the wage moves further from output as the output of a job increases. Examining exit rates at a wage of $\$ 15.00$ for example, is the same as output of 15 in the pair-wise efficient contract, while it corresponds to output of much more that 15 in 
the inefficient contract. Thus, that the exit rates for the pair-wise efficient contract eventually lie above those for the inefficient contract, when plotted by wages, highlights both the nature of the inefficiency in wages, and that this inefficiency grows as the output of the job increases.

Figure 4: Employed Exit Rate by Output and Wage - Men \& Women
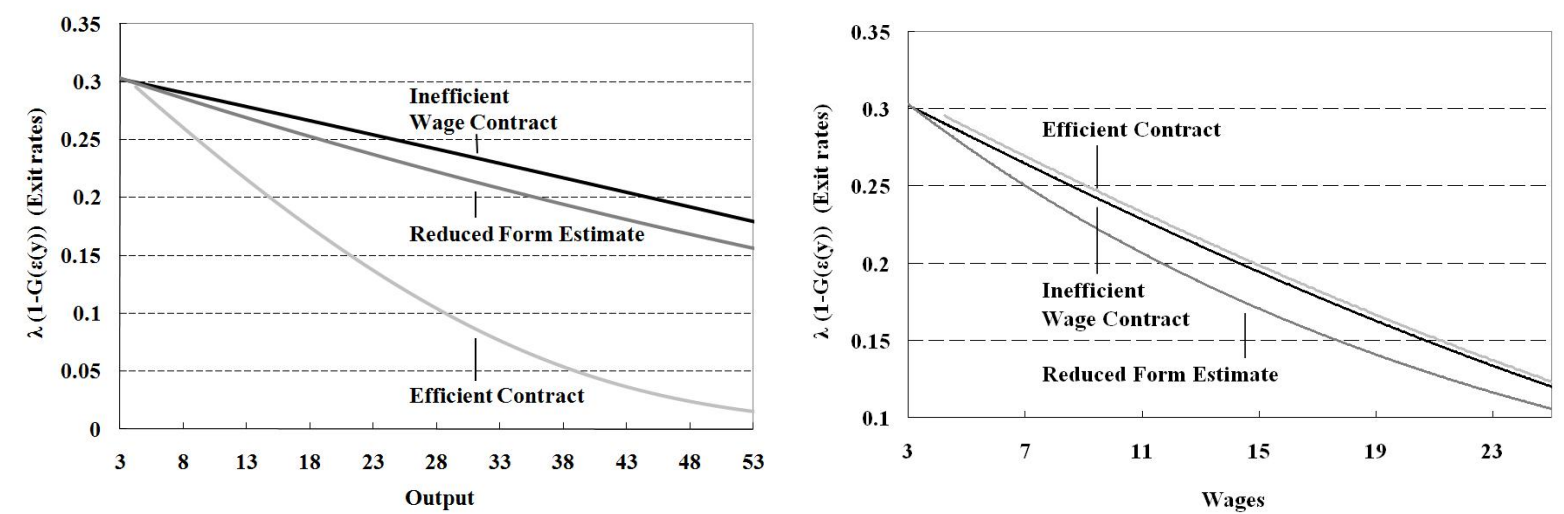

In addition to exit rates, we have plotted in Figure 5 the optimal hiring fee $(\phi)$ against each job's output, using the parameters from Specification I. Note, it is convex, zero at the reservation wage (since the firm is earning no profits), and increasing. Overall, the size of the hiring fee is not extremely high.

Figure 5: Pair-wise Efficient Hiring Fee

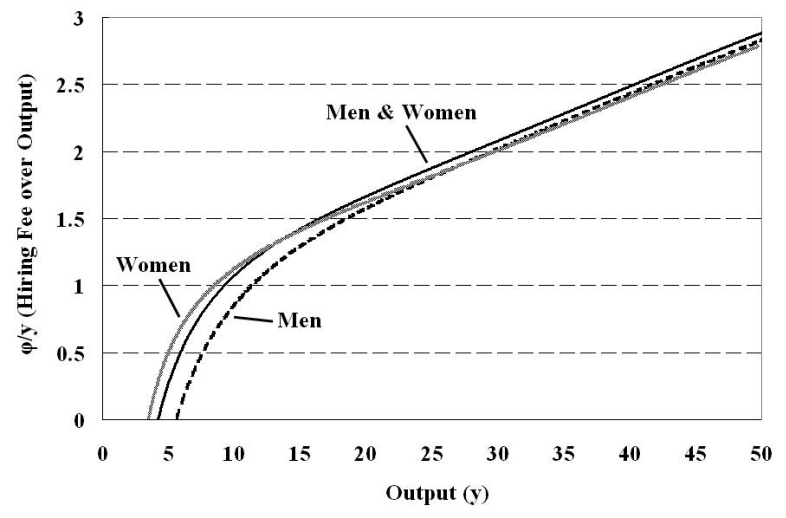




\section{Robustness}

In this section we discuss potential issues in the estimation and robustness checks of our results with respect to these concerns.

First, the welfare comparisons between the inefficient and pair-wise efficient contract are affected by our imposing the Hosios condition, i.e., $\eta=\beta$. However, as we discussed in Section 2.2.3, this may not be efficient (in terms of maximizing welfare). Albrecht et al. (2010) show that in a model with endogenous labor market entry, when the Hosios condition is imposed too many vacancies are created, or labor force participation is too high. This essentially works "against" the inefficiency we describe, whereby labor force participation remains too low. Thus, there exists the possibility that the entry inefficiency helps the exit inefficiency, and the constant wage contract may actually represent a welfare improvement from the pair-wise pair-wise efficient contract. To explore the issue, we vary $\eta$ and calculate the model in each case. ${ }^{13}$ At the extreme we found the welfare gain from switching from the inefficient contract to the pair-wise efficient contract is potentially smaller but still positive. For example, when using the male and female sample, we found restricting $\eta=0.95$ results in a welfare gain of $2.2 \%$ rather than $3.5 \%$ while restricting $\eta=0.05$ results in a welfare gain of $4.4 \%$. For women, who had a smaller welfare gain, the positive increase goes from $1.25 \%$ to $0.92 \%$. Therefore, while the restriction does not change our main results, it does affect the magnitude of the inefficiency. A further exploration of efficiency along the entry margin in a model with this exit inefficiency is an interesting direction for future research.

Second, we have estimated the model without the employed wage data using flow sampling. This approach can highlight any discrepancies between the duration data and the proportion of individuals in each state. At the same time, Figure 3 suggests the estimation is affected by the peak in the employed hourly wage at $\$ 8$. Therefore, it seems appropriate to ask what happens if this data is excluded. The answer is the results were unaffected when using a flow sampling approach without the employed wage data.

Third, the wage distribution is key in testing the two models and the distribution relies heavily on the parametric specification of the productivity distribution. We have provided the results using the log-normal distribution as it is the standard in the literature and can easily be compared. How-

\footnotetext{
${ }^{13}$ Again, estimating $\eta$ using the approaches found in Flinn (2006) or Engelhardt \& Rupert (2011) would not change the likelihood ratio test used to reject the pair-wise efficient contract. We have not used these techniques to estimate $\eta$ because of the restrictive parametric assumptions required and the nature of the data used.
} 
ever, we have run the model with a Weibull productivity distribution (and thus the other commonly used productivity distribution - the exponential). The results are presented in Table 5. When using the Weibull distribution, we find nearly identical results regarding the parameter estimates as well as the level of significance at which pair-wise efficiency can be rejected.

Finally, workers who make job-to-job transition introduces an externality similar to the one we study for the employment to non-participation transition. To the extent that we observe such transitions in the data, it remains possible that ignoring this feature in our model could affect the results. First, since we observe job-to-job transitions in the data, we need to have a method to handle these cases within the context of our model. Our solution is when we observe an individual transition from employment to employment, we assume they spend a short time, less than the interval of measure, unemployed. Thus, in Table 1, the employment to unemployment transitions also include job-to-job transitions. Given the issue, we have estimated the model after removing all observations who make a job-to-job transition. The results are in Table 6.

We find that this does not affect our main results. Specifically, the likelihood ratio test statistic is 97.6 with a p-value roughly equal to zero. The analysis indicates that while job-to-job transitions occur and are potentially interesting, their exclusion does not alter our conclusions regarding the relative fit of each model. Moreover, in the NLSY data, transitions from employment to nonparticipation occur more frequently than job-to-job transitions. Specifically, we find that among all employed individuals (for whom we observe a complete employment spell), $45 \%$ transition to non-participation, while only $33 \%$ transition from job-to-job, and even fewer, $22 \%$ transition to unemployment. Thus, while job-to-job transitions represent an interesting and important feature to consider when analyzing wage contracts, we focus on the empirically more likely transition of employment to non-participation; moreover, we find that excluding job-to-job transitions does not affect our results.

\section{Conclusion}

We develop an equilibrium search model capable of accounting for observed transitions in and out of the labor market. When individuals receive idiosyncratic shocks affecting the value of nonmarket activity, an employment contract consisting of only a constant wage remains inefficient. In the pair-wise efficient employment contract, individuals receive a wage equal to the productivity 


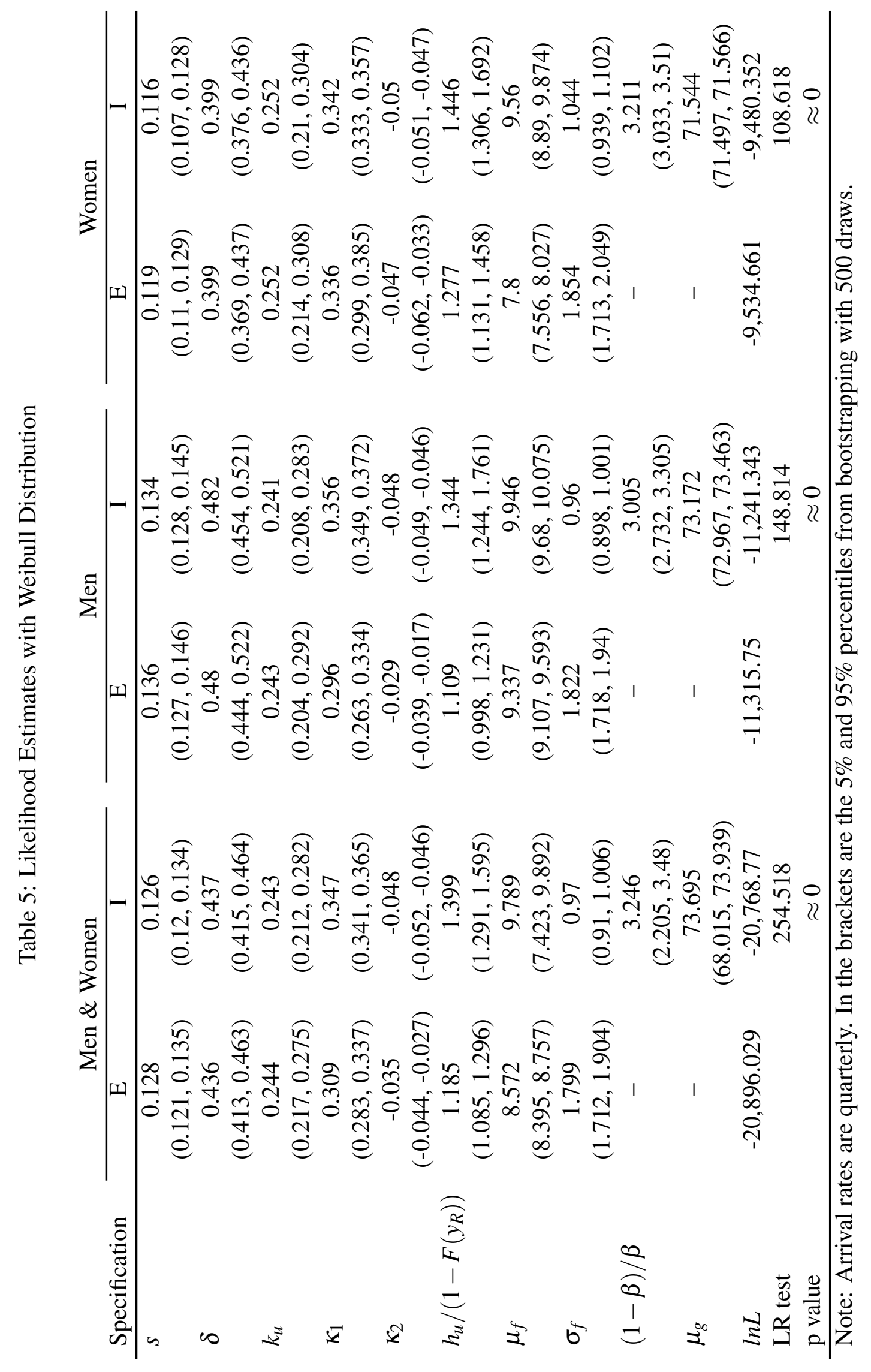


Table 6: Likelihood Estimates without Job-to-Job Transitions

\begin{tabular}{lcccccc}
\hline \multirow{2}{*}{ Specification } & \multicolumn{2}{c}{ Men \& Women } & \multicolumn{2}{c}{ Men } & \multicolumn{2}{c}{ Women } \\
\hline$s$ & $\mathrm{E}$ & $\mathrm{I}$ & $\mathrm{E}$ & $\mathrm{I}$ & $\mathrm{E}$ & $\mathrm{I}$ \\
$\delta$ & 0.078 & 0.077 & 0.088 & 0.087 & 0.066 & 0.065 \\
$k_{u}$ & 0.398 & 0.398 & 0.437 & 0.438 & 0.364 & 0.364 \\
$\kappa_{1}$ & 0.304 & 0.303 & 0.301 & 0.3 & 0.313 & 0.313 \\
$\kappa_{2}$ & 0.322 & 0.354 & 0.317 & 0.363 & 0.34 & 0.345 \\
$h_{u} /\left(1-F\left(y_{R}\right)\right)$ & -0.037 & -0.047 & -0.031 & -0.046 & -0.048 & -0.05 \\
$\mu_{f}$ & 0.773 & 0.787 & 0.737 & 0.746 & 0.823 & 0.842 \\
$\sigma_{f}$ & 2.039 & 2.331 & 2.105 & 2.502 & 1.968 & 2.135 \\
$(1-\beta) / \beta$ & 0.398 & 0.636 & 0.403 & 0.665 & 0.38 & 0.578 \\
$\mu_{g}$ & - & 2.263 & - & 2.882 & - & 1.617 \\
$\ln L$ & - & 71.484 & - & 71.475 & - & 71.498 \\
$\mathrm{LR}$ test & $-14,967.76$ & $-14,918.96$ & $-7,914.75$ & $-7,890.48$ & $-7,014.94$ & $-6,989.00$ \\
$\mathrm{p}$ value & & 97.6 & & 48.55 & & 51.88 \\
& & $\approx 0$ & & $\approx 0$ & & $\approx 0$ \\
\hline
\end{tabular}

of the match, and pay a hiring fee upon its formation. The analysis in this paper highlights the difficulties facing researchers attempting to study labor market outcomes, wage contracts, and the implications of various policies on these outcomes. Based on our conclusions, the pair-wise efficient employment contract does not appear to be consistent with the data, suggesting a role for labor market policies, specifically those aimed at reducing the flow of individuals from employment to inactivity.

In this paper we do not discuss potential policies that could provide a structural shift from the observed equilibrium to one more closely resembling the pair-wise efficient contract. These alternatives provide an interesting direction for future research. For example, a tax and transfer scheme could be designed in a manner that produces wage contracts similar to the pair-wise efficient contract. In addition, future research should focus on determining what factors may be preventing the efficient outcome from occurring. 


\section{A Appendix}

Proof of Lemma 1 According to Nash's axioms, $(\phi, w)$ must be pairwise Pareto-efficient. Since the up-front payment $\phi$ allows the worker and the firm to transfer utility perfectly, the wage, $w$, must be chosen to maximize the total surplus of the match. Define $\mathscr{S}(y) \equiv \mathscr{V}_{e}(y)-\mathscr{V}_{u}+\mathscr{V}_{f}(y)$, which represents the total surplus of a match (recall $\mathscr{V}_{v}=0$ ). From (2) and (10),

$$
r \mathscr{S}(y)=y-r \mathscr{V}_{u}-s \mathscr{S}(y)+\lambda_{e} \int_{\varepsilon_{e}}^{\bar{\varepsilon}}\left[\varepsilon-\mathscr{S}-\left(\mathscr{V}_{u}-\mathscr{V}_{o}\right)\right] d G(\varepsilon)
$$

Notice, the surplus of the match is maximized if

$$
\varepsilon_{e}(y)=\left(\mathscr{S}(y)+\mathscr{V}_{u}-\mathscr{V}_{o}\right)=\left(\mathscr{V}_{e}(y)+\mathscr{V}_{f}(y)-\mathscr{V}_{o}\right)
$$

The comparison of (7) and (35) shows that the match surplus is maximized iff $\mathscr{V}_{f}=0$. From (10), $\mathscr{V}_{f}=0$ requires $w=y$. Finally, the first-order condition of (12) with respect to $\phi$ yields (14).

\section{Proof of Lemma 2:}

Proof. Finding an equilibrium is a fixed point problem in $\theta$. Given a value of $\theta$, equations (23) and (24) determine $\varepsilon_{u}$ and $\varepsilon_{e}(y)$, as functions of $\theta$. Then, (25) can be calculated, and depending on its sign, a new value of $\theta$ determined. The process continues until (25) holds. From (25), define the operator $\Gamma(\theta)$ as

$$
\Gamma(\theta)=\int_{y_{R}}\left\{\chi y+(1-\chi) w(y)-b-\frac{\beta \theta \gamma}{1-\beta}+\lambda_{e} h\left[\varepsilon_{e}(y)\right]-\lambda_{u} h\left(\varepsilon_{u}\right)\right\} d F(y)-\frac{(r+s) \gamma}{(1-\beta) q(\theta)}
$$

We begin with the pair-wise efficient contract case, $\chi=1$. The idea of the proof is to show that there exists $\theta^{\star}>0$ such that $\Gamma\left(\theta^{\star}\right)=0$. This is accomplished by showing $\Gamma(0)<0$ and $\Gamma(\infty)>0$. Towards this end, consider $\lim _{\theta \rightarrow \infty} \Gamma(\theta)$. As a matter of notation, denote $\lim _{\theta \rightarrow 0} \varepsilon_{e}(y) \equiv \varepsilon_{e}^{0}(y)$, $\lim _{\theta \rightarrow \infty} \varepsilon_{e}(y) \equiv \varepsilon_{e}^{\infty}(y), \lim _{\theta \rightarrow 0} \varepsilon_{u} \equiv \varepsilon_{u}^{0}, \lim _{\theta \rightarrow \infty} \varepsilon_{u} \equiv \varepsilon_{u}^{\infty}, \lim _{\theta \rightarrow 0} y_{R} \equiv y_{R}^{0}$, and $\lim _{\theta \rightarrow \infty} y_{R} \equiv y_{R}^{\infty}$. Notice, given the properties of $q(\theta), \lim _{\theta \rightarrow \infty} \frac{\gamma}{q(\theta)}=-\infty$, so that $\lim _{\theta \rightarrow \infty} \Gamma(\theta)=-\infty$. Now consider $\lim _{\theta \rightarrow 0} \Gamma(\theta)$. The sign of this limit is determined by

$$
\int_{y_{R}^{0}}\left\{y-b+\lambda_{e} h\left[\varepsilon_{e}^{0}(y)\right]-\lambda_{u} h\left(\varepsilon_{u}^{0}\right)\right\} d F(y)
$$

Since $y>b, \forall y \geq y_{R}$ (by assumption), we need only to show $\lambda_{e} h\left(\varepsilon_{e}^{0}(y)\right)-\lambda_{u} h\left(\varepsilon_{u}^{0}\right)>0$. To do so, suppose instead that $\lim _{\theta \rightarrow 0} \Gamma(\theta) \leq 0$, which implies (21) does not hold, or more specifically (using 
$\left.\mathscr{V}_{e}(y)-\mathscr{V}_{u}=\varepsilon_{e}(y)-\varepsilon_{u}\right)$

$$
\begin{gathered}
\frac{1-\beta}{\beta}\left(\int_{y_{R}^{0}} \varepsilon_{e}^{0}(y) d F(y)-\left[1-F\left(y_{R}^{0}\right)\right] \varepsilon_{u}^{0}\right) \leq \frac{\gamma}{q(\theta)}=0 \\
\Rightarrow \varepsilon_{u}^{0} \geq \frac{1}{1-F\left(y_{R}^{0}\right)} \int_{y_{R}^{0}} \varepsilon_{e}^{0}(y) d F(y) \\
\Rightarrow-h\left(\varepsilon_{u}^{0}\right)>-h\left[\int_{y_{R}} \varepsilon_{e}^{0}(y) d F(y)\right]
\end{gathered}
$$

where the last line follows from the properties of $h(\cdot)$ and that $\left[1-F\left(y_{R}\right)\right]<1$. Further, since $\lambda_{e} \geq \lambda_{u}$ by assumption, we have

$$
\begin{gathered}
\int_{y_{R}^{0}}\left\{\lambda_{e} h\left(\varepsilon_{e}^{0}(y)\right)-\lambda_{u} h\left(\varepsilon_{u}^{0}\right)\right\} d F(y)>\lambda_{u} \int_{y_{R}^{0}}\left\{h\left(\varepsilon_{e}^{0}(y)\right)-h\left[\int_{y_{R}^{0}} \varepsilon_{e}^{0}(y) d F(y)\right]\right\} d F(y) \\
=\lambda_{u}\left[\int_{y_{R}^{0}} h\left(\varepsilon_{e}^{0}(y)\right) d F(y)-\left[1-F\left(y_{R}^{0}\right)\right] h\left[\int_{y_{R}^{0}} \varepsilon_{e}^{0}(y) d F(y)\right]\right]>0
\end{gathered}
$$

where the last inequality follows from the fact that $\left[1-F\left(y_{R}\right)\right]<1$ and that the convexity of $h(x)$ means Jensen's inequality implies $\int_{y_{R}^{0}} h\left(\varepsilon_{e}^{0}(y)\right) d F(y)>h\left[\int_{y_{R}^{0}} \varepsilon_{e}^{0}(y) d F(y)\right]$. However, this fact implies that

$$
\lim _{\theta \rightarrow 0} \Gamma(\theta)>0
$$

a contradiction. Since $\Gamma(0)>0, \Gamma(\infty)=-\infty$, and the continuity of $\Gamma(\theta)$, the Intermediate Value Theorem implies there exists $\theta^{\star}>0$ such that $\Gamma\left(\theta^{\star}\right)=0$.

Now consider the case of constant wage contracts, $\chi=0$. Recall, $w_{R}=y_{R}>b$, which implies $w(y)>b, \forall y$; thus, the same proof above applies to this case.

The following Lemma is needed in order to show the uniqueness of equilibria.

Lemma 3 The reservation wage satisfies $\frac{d y_{R}}{d \theta}>0$.

Proof. Since at the reservation wage, $\mathscr{V}_{e}\left(y_{R}\right)=\mathscr{V}_{u}$, and for both $\chi=1$ and $\chi=0, w\left(y_{R}\right)=y_{R}$, using (1) and (2) we can write

$$
y_{R}=b+\frac{\beta \gamma \theta}{1-\beta}+\left(\lambda_{u}-\lambda_{e}\right) h\left(\varepsilon_{u}\right)
$$

where we also use the fact that $\varepsilon_{e}\left(y_{R}\right)=\varepsilon_{u}$. Then, differentiating (39) with respect to $\theta$ gives

$$
\frac{d y_{R}}{d \theta}=\frac{\beta \gamma}{1-\beta}+\left(\lambda_{u}-\lambda_{e}\right) h^{\prime}\left(\varepsilon_{u}\right) \frac{d \varepsilon_{u}}{d \theta}
$$


Since $\lambda_{e} \geq \lambda_{u}$ by assumption, $h^{\prime}(x)<0$ combined with $\frac{d \varepsilon_{u}}{d \theta}>0$ (from (23) and $\lambda_{u} \geq \lambda_{o}$ ) implies this expression is strictly positive.

We can now show the following result regarding the uniqueness of equilibria.

Lemma 4 If $\frac{d \varepsilon_{e}(y)}{d \theta} \leq \frac{d \varepsilon_{u}}{d \theta}, \forall y \geq y_{R}$, then there exists a unique equilibrium.

Proof. Given a value of $\theta$, equations (23) and (24) determine unique values of $\varepsilon_{e}(y)$ and $\varepsilon_{u}$. Given these, $\Gamma(\theta)$ can be computed. To verify that there exits a unique $\theta^{\star}>0$ such that $\Gamma\left(\theta^{\star}\right)=0$, it suffices to show that $\Gamma(\theta)$ is strictly decreasing in $\theta$. Since $\forall y \geq y_{R}, \mathscr{V}_{e}(y) \geq \mathscr{V}_{u}$, the integrand in (36) must be positive for all values of $\theta>0$. The key to the proof is to show that when $\theta$ increases, the integrand must decrease for all $y$, and $y_{R}$ increases. These two facts (combined with the positive integrand) imply the desired result. Define the function $z(y ; \theta)=$ $\chi y+(1-\chi) w(y)-b+\frac{\beta \theta \gamma}{1-\beta}+\lambda_{e} h\left[\varepsilon_{e}(y)\right]-\lambda_{u} h\left(\varepsilon_{u}\right)$. Then, consider $\frac{d z(y ; \theta)}{d \theta}$, which is given by

$$
\frac{d z(y ; \theta)}{d \theta}=(1-\chi) \frac{d w(y)}{d \theta}-\frac{\beta \gamma}{1-\beta}+\lambda_{e} h^{\prime}\left[\varepsilon_{e}(y)\right] \frac{d \varepsilon_{e}(y)}{d \theta}-\lambda_{u} h^{\prime}\left(\varepsilon_{u}\right) \frac{d \varepsilon_{u}}{d \theta}
$$

Now, differentiating (23) with respect to $\theta$ and rearranging gives

$$
\lambda_{u} h^{\prime}\left(\varepsilon_{u}\right) \frac{d \varepsilon_{u}}{d \theta}=\left(r+\lambda_{0}\right) \frac{d \varepsilon_{u}}{d \theta}-\frac{\beta \gamma}{1-\beta}+\lambda_{0} h^{\prime}\left(\varepsilon_{u}\right) \frac{d \varepsilon_{u}}{d \theta}
$$

Similarly, differentiating (24) with respect to $\theta$ and rearranging yields

$$
\lambda_{e} h^{\prime}\left[\varepsilon_{e}(y)\right] \frac{d \varepsilon_{e}(y)}{d \theta}=(r+s) \frac{d \varepsilon_{e}(y)}{d \theta}-\left(s-\lambda_{0}\right) \frac{d \varepsilon_{u}}{d \theta}+\lambda_{0} h^{\prime}\left(\varepsilon_{u}\right) \frac{d \varepsilon_{u}}{d \theta}-(1-\chi) \frac{d w(y)}{d \theta}
$$

Subtracting (41) from (42) and plugging into (40) gives

$$
\frac{d z(y ; \theta)}{d \theta}=(r+s)\left[\frac{d \varepsilon_{e}(y)}{d \theta}-\frac{d \varepsilon_{u}}{d \theta}\right]
$$

which by assumption is negative. Thus, using this fact and Lemma 3, for any $\theta_{1}<\theta_{2}$,

$$
\begin{aligned}
& \int_{y_{R}^{1}}\left\{\chi y+(1-\chi) w(y)-b-\frac{\beta \theta_{1} \gamma}{1-\beta}+\lambda_{e} h\left[\varepsilon_{e}^{1}(y)\right]-\lambda_{u} h\left(\varepsilon_{u}^{1}\right)\right\} d F(y) \\
> & \int_{y_{R}^{2}}\left\{\chi y+(1-\chi) w(y)-b-\frac{\beta \theta_{1} \gamma}{1-\beta}+\lambda_{e} h\left[\varepsilon_{e}^{1}(y)\right]-\lambda_{u} h\left(\varepsilon_{u}^{1}\right)\right\} d F(y) \\
> & \int_{y_{R}^{2}}\left\{\chi y+(1-\chi) w(y)-b-\frac{\beta \theta_{2} \gamma}{1-\beta}+\lambda_{e} h\left[\varepsilon_{e}^{2}(y)\right]-\lambda_{u} h\left(\varepsilon_{u}^{2}\right)\right\} d F(y)
\end{aligned}
$$


Thus, we have shown $\Gamma(\theta)$ to be strictly decreasing, and therefore there exists a unique $\theta^{\star}$ such that $\Gamma\left(\theta^{\star}\right)=0$. Finally, given unique values of $\varepsilon_{u}, \varepsilon_{e}(y)$, and $\theta$, equations (27)-(28) determine unique values of $n_{e}(y), n_{u}$, and $n_{o}$.

\section{Proof of Proposition 1:}

Proof. From Lemma 2, an active equilibrium exists. The key to the proof of uniqueness is to show that for all $y \geq y_{R}, \frac{d \varepsilon_{e}(y)}{d \theta}<\frac{d \varepsilon_{u}}{d \theta}$. Then, Lemma 4 gives the desired result. From equation (24) we can write

$$
(r+s) \varepsilon_{e}(y)=y-x+\lambda_{e} h\left(\varepsilon_{e}(y)\right)+\left(s-\lambda_{0}\right) \varepsilon_{u}-\lambda_{0} h\left(\varepsilon_{u}\right)
$$

Then, differentiating with respect to $\theta$ yields

$$
\frac{d \varepsilon_{e}(y)}{d \theta}=\left(\frac{s-\lambda_{0}\left[1+h^{\prime}\left(\varepsilon_{u}\right)\right]}{r+s-\lambda_{e} h^{\prime}\left[\varepsilon_{e}(y)\right]}\right) \frac{d \varepsilon_{u}}{d \theta}
$$

We can now show that the ratio multiplying $\frac{d \varepsilon_{u}}{d \theta}$ is less than 1 . Given the definition of $h(x)$, $h^{\prime}(x)=G(x)-1$. Then, we have the following:

$$
\begin{gathered}
-\lambda_{0} G\left(\varepsilon_{u}\right)<r-\lambda_{e}\left[G\left(\varepsilon_{e}(y)\right)-1\right] \\
\Rightarrow s-\lambda_{0} G\left(\varepsilon_{u}\right)<r+s-\lambda_{e}\left[G\left(\varepsilon_{e}(y)\right)-1\right] \\
=s-\lambda_{0}\left[1+h^{\prime}\left(\varepsilon_{u}\right)\right]<r+s-\lambda_{e} h^{\prime}\left(\varepsilon_{e}(y)\right)
\end{gathered}
$$

and therefore, $\frac{d \varepsilon_{e}(y)}{d \theta}<\frac{d \varepsilon_{u}}{d \theta}$.

Now we can prove the sufficient condition for uniqueness of equilibrium in the constant wage contract case.

\section{Proof of Proposition 2.}

Proof. Assume that the support of $F(y)$ is bounded, $[\underline{y}, \bar{y}] .{ }^{14}$ The proof proceeds in a similar manner to the $\chi=1$ case. Specifically, we need to show that $\Gamma(\theta)$ is strictly decreasing in $\theta$. From Lemma 4 , this occurs when $\frac{d \varepsilon_{e}(y)}{d \theta} \leq \frac{d \varepsilon_{u}}{d \theta}$. To analyze this relationship in the $\chi=0$ case, differentiate (24) with respect to $\theta$ (after plugging in for $w(y)$ ), which after some simplification

\footnotetext{
${ }^{14}$ This assumption is not necessary to prove the result, but simplifies the proof by allowing use of Leibniz formula, and the main idea of the proof is more transparent. Alternatively, the derivative could be calculated directly using limits.
} 
yields

$$
\begin{gathered}
\frac{d \varepsilon_{e}(y)}{d \theta}\left[r+s-\lambda_{e} h^{\prime}\left(\varepsilon_{e}(y)\right)+\frac{1-\beta}{\beta}\left(r+s+\lambda_{e}\left[1-G\left(\varepsilon_{e}(y)\right)\right]-\lambda_{e}\left(\varepsilon_{e}(y)-\varepsilon_{u}\right) g\left(\varepsilon_{e}(y)\right)\right)\right]= \\
\frac{d \varepsilon_{u}}{d \theta}\left[s-\lambda_{o}\left(1+h^{\prime}\left(\varepsilon_{u}\right)\right)+\frac{1-\beta}{\beta}\left(r+s+\lambda_{e}\left[1-G\left(\varepsilon_{e}(y)\right)\right]\right)\right]
\end{gathered}
$$

Notice, that when $y=y_{R}$, since $\varepsilon_{e}\left(y_{R}\right)=\varepsilon_{u}$, the arguments in the proof of Proposition 1 above can be used to show that $\frac{d \varepsilon_{e}\left(y_{R}\right)}{d \theta}<\frac{d \varepsilon_{u}}{d \theta}$. Since $\frac{d \varepsilon_{e}(y)}{d \theta}$ is continuous in $y$, there exists a $\Delta>0$ such that $\frac{d \varepsilon_{e}(y)}{d \theta} \leq \frac{d \varepsilon_{u}}{d \theta}$ for all $y \in\left[y_{R}, y_{R}+\Delta\right)$. Thus, we can set $\hat{y}=y_{R}+\Delta$. Now, recall the key to showing $\Gamma(\theta)$ is strictly decreasing is the term $\int_{y_{R}}^{\bar{y}} z(y ; \theta) f(y) d y$. Using Leibniz rule, we can write

$$
\begin{aligned}
& \frac{\partial}{\partial \theta} \int_{y_{R}(\theta)}^{\bar{y}} z(y ; \theta) f(y) d y=\int_{y_{R}(\theta)}^{\bar{y}} \frac{\partial}{\partial \theta} z(y ; \theta) f(y) d y-\frac{d y_{R}(\theta)}{d \theta} z\left(y_{R}(\theta) ; \theta\right) f\left(y_{R}\right) \\
& =\int_{y_{R}(\theta)}^{\hat{y}} \frac{\partial}{\partial \theta} z(y ; \theta) f(y) d y+\int_{\hat{y}}^{\bar{y}} \frac{\partial}{\partial \theta} z(y ; \theta) f(y) d y-\frac{d y_{R}(\theta)}{d \theta} z\left(y_{R}(\theta) ; \theta\right) f\left(y_{R}\right)
\end{aligned}
$$

Since Lemma 3 implies $\frac{d y_{R}(\theta)}{d \theta}>0, z\left(y_{R} ; \theta\right) \geq 0$ implies the third term on the R.H.S. of (47) is positive, and the sign of this derivative depends on the term $\int_{\hat{y}}^{\bar{y}} \frac{\partial}{\partial \theta} z(y ; \theta) f(y) d y$. If $\hat{y}=\bar{y}$, we are done. In the case of $\hat{y}<\bar{y}$, we can select an appropriate $A$, so that $\forall y \geq \hat{y}, f(y) \leq A$ implies

$$
\int_{\hat{y}}^{\bar{y}} \frac{\partial}{\partial \theta} z(y ; \theta) f(y) d y<-\left[\int_{y_{R}(\theta)}^{\bar{y}} \frac{\partial}{\partial \theta} z(y ; \theta) f(y) d y\right]+\frac{d y_{R}(\theta)}{d \theta} z\left(y_{R}(\theta) ; \theta\right) f\left(y_{R}\right)
$$

That is, if $f(y)$ is sufficiently small for $y \geq \hat{y}$, the integral $\int_{\hat{y}}^{\bar{y}} \frac{\partial}{\partial \theta} z(y ; \theta) f(y) d y$, while positive, is not "too" large, and $\frac{\partial}{\partial \theta} \int_{y_{R}(\theta)}^{\bar{y}} z(y ; \theta) f(y) d y$ is still negative. Thus, $\frac{\partial}{\partial \theta} \Gamma(\theta)<0$, and there exists unique $\theta^{*}$ solving $\Gamma\left(\theta^{*}\right)=0$. Given this unique value of $\theta^{\star}$, equations (23) and (24) determine unique values of $\varepsilon_{e}(y)$ and $\varepsilon_{u}$. Finally, given unique values of $\varepsilon_{u}, \varepsilon_{e}(y)$, and $\theta$, equations (27)-(28) determine unique values of $n_{e}(y), n_{u}$, and $n_{o}$. 


\section{References}

Albrecht, J. W., Navarro, L. \& Vroman, S. B. (2010), 'Efficiency in a Search and Matching Model with Endogenous Participation', Economic Letters 106, 48-50.

Bonilla, R. \& Burdett, K. (2005), Bargaining, On-the-Job Search and Labour Market Equilibrium, Henning Bunzel and Bent J. Christensen and George R. Neumann and Jean-Marc Robin edn, North-Holland, Amsterdam.

Bontemps, C., Robin, J.-M. \& van den Berg, G. J. (2000), 'Equilibrium Search with Continuous Productivity Dispersion: Theory and Nonparametric Estimation', International Economic Review 41, 305-58.

Bowlus, A. J., Kiefer, N. M. \& Neumann, G. R. (1995), 'Estimation of Equilibrium Wage Distributions with Heterogeneity', Journal of Applied Econometrics 10, S119-S131.

Bowlus, A. J. \& Neumann, G. R. (2005), The Job Ladder, Henning Bunzel and Bent J. Christensen and George R. Neumann and Jean-Marc Robin edn, North-Holland, Amsterdam.

Carmichael, H. L. (1990), 'Efficiency Wage Models of Unemployment - One View', Economic Inquiry 28, 269-295.

Engelhardt, B., Rocheteau, G. \& Rupert, P. (2008), 'Crime and the Labor Market: A Search Model with Optimal Contracts', Journal of Public Economics 92, 1876-1891.

Engelhardt, B. \& Rupert, P. (2011), Competitive Search: A Test for Direction, Efficiency, and Submarkets. Working Paper.

Flinn, C. \& Heckman, J. (1982), 'New Methods for Analyzing Structural Models of Labor Force Dynamics', Journal of Econometrics 18, 115-168.

Flinn, C. J. (2006), 'Minimum Wage Effects on Labor Market Outcomes Under Search, Matching, and Endogeneous Contact Rates', Econometrica 74, 1013-1062.

Flinn, C. \& Mabli, J. (2008), On-the-Job Search, Minimum Wages, and Labor Market Outcomes in an Equilibrium Bargaining Framework. Institute for Research on Poverty, Discussion Paper no. 1337-08. 
Garibaldi, P. \& Wasmer, E. (2005), 'Equilibrium Search Unemployment, Endogenous Participation, and Labor Market Flows', Journal of the European Economic Association 3, 851-882.

Gautier, P. A., Moraga-Gonzalez, J. L. \& Wolthoff, R. P. (2009), Structural Estimation of Search Intensity: Do Non-Employed Workers Search Enough? Mimeo.

Hagedorn, M. \& Manovskii, I. (2008), 'The Cyclical Behavior of Equilibrium Unemployment and Vacancies Revisited', The American Economic Review 98, 1692-1706.

Hosios, A. J. (1990), 'On the Efficiency of Matching and Related Models of Search Unemployment', Review of Economic Studies 57, 279-298.

Lancaster, T. (1990), The Econometric Analysis of Transition Data, Cambridge University Press, New York, NY.

Menzio, G. \& Shi, S. (2011), Efficient Search on the Job and the Business Cycle. Mimeo.

Pissarides, C. A. (2000), Equilibrium Unemployment Theory, MIT Press, Cambridge, MA.

Pries, M. \& Rogerson, R. (2004), Search Frictions and Labor Market Participation. Working Paper.

Shimer, R. (2005a), 'On-the-job Search and Strategic Bargaining', European Economic Review 50, 811-830.

Shimer, R. (2005b), 'The Cyclical Behavior of Equilibrium Unemployent and Vacancies', American Economic Review 95, 25-49.

Stevens, M. (2004), 'Wage-tenure contracts in a frictional labor market: Firms' strategies for recruitment and retention', Review of Economic Studies 71, 535-551.

van den Berg, G. J. \& Ridder, G. (1998), ‘An Empirical Equilibrium Search Model of the Labor Market', Econometrica 66, 1183-1221.

Wolpin, K. I. (1987), 'Estimating a Structural Search Model: The Transition from School to Work', Econometrica 55.

Yip, C. S. (2003), Job Search and Labor Force Participation in Equilibrium: Theory and Estimation. Manuscript. 